ZOOLOGIA 31 (4): 343-360, August, 2014

http://dx.doi.org/10.1590/S1984-46702014000400006

\title{
Bird diversity in the Serra do Aracá region, northwestern Brazilian Amazon: preliminary check-list with considerations on biogeography and conservation
}

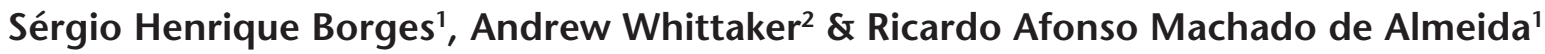

\author{
${ }^{1}$ Fundação Vitória Amazônica. Rua Estrela D'Alva 146, Loteamento Parque Morada do Sol, Aleixo, 69060-093 Manaus, AM, \\ Brazil.E-mail: sergio@fva.org.br \\ ${ }^{2}$ Museu Paraense Emílio Goeldi. Caixa Postal 399, 66040-170 Belém, PA, Brazil. E-mail: andrew@birdingbraziltours.com
}

\begin{abstract}
We inventoried the birds from Serra do Aracá region, state of Amazonas. The region encompasses a high diversity of vegetation types, including white sand forests and campinas, terra firme and flooded forests, montane forests and tepuis. We recorded 416 bird taxa in 69 families through captures with mist nets, tape recording of bird voices, and collection of voucher specimens. A large proportion of them (61\%) were recorded in a single vegetation type. Qualitative estimates suggest that approximately 580 bird species occur in the region. The avifauna of the Aracá region has a mixed biogeographic composition, with species typical of both margins of the Rio Negro occurring sympatrically. Additionally, species whose distributions are restricted to three areas of endemism for Amazonian birds (Imeri, Guiana and Pantepui) were recorded in the region. Rare landscapes in the Brazilian Amazon are found in the Serra do Aracá region. Additionally, we recorded endemic and rare birds, highlighting the value of the region for conservation. The Serra do Aracá State Park officially protects montane forests, terra firme forests and tepuis. We suggest that the large extension of white sand campinas and igapó forests at the southern portion of Serra do Aracá should be also preserved in order to improve the representation of the rich natural heritage of the region.
\end{abstract}

KEY WORDS. Amazon biodiversity; Amazonas; Rio Branco; Rio Negro; tepuis; white sand campinas.

The biogeographic distribution of the Amazonian avifauna is complex, and includes geographic gradients of species diversity and the influence of rivers in the delimitation of areas of endemism (Cracraft 1985, Haffer 1992, Rahbek \& Graves 2001, Silva et al. 2005, CoHn-Haft et al. 2007a, b). Although the major geographic patterns of bird distribution in Amazonia have been identified since the late 1960's (HAFFER 1969, 1974), recent studies have documented new areas of endemism, areas of biogeographic transition and large scale turnover of species composition in flooded forests (CoHN-Haft et al. 2007b, NAKA 2011, Borges \& SiLva 2012). These studies have demonstrated that larger scale inventories are useful for understanding the geographic patterns of bird distribution within the Amazon basin.

Although recent inventories have elucidadet the distribution of birds in the Amazon (see papers in SANTOS \& Aleixo 2011), several locations remain poorly investigated (Oren \& AlbuquerQUE 1991, OREN 2001). The avifauna of the region located between the Branco and Negro rivers in the state of Amazonas, for example, remains poorly studied despite recent inventories (NAKA et al. 2006, NAKA et al. 2007). This region includes rare ecosystems of the Amazon such as white sand campinas, montane forests and tepuis (Prance \& Johnson 1992, Santos et al. 1993). Moreover, this region is located between rivers that have a clear influence on the distribution of birds and other taxa (AYres \& Clutton-Brock 1992, Borges 2007, NAKA 2011).

We provide a preliminary check-list of the birds from the Serra do Aracá region, which is located in the Rio Negro/ Rio Branco interfluve, Northwestern Brazilian Amazon. Additionally, we perform qualitative analysis of the bird fauna, to understand how habitat heterogeneity and geographical context influence species distribution. And finally, we consider the effectivennes of the Serra do Aracá State Park to protect a representative sample of the regional diversity of bird species and environments.

\section{MATERIAL AND METHODS}

Birds were sampled in the major habitats of the Serra do Aracá and the valleys of the Aracá and Demini rivers, at the Negro/Branco interfluve in 2004 (December), 2006 to 2008 (July/August) and 2010 (July/August) years. During the expeditions we held nearly 55 field days, which were distributed among 25 sites throughout the region (Fig. 1), though not evenly. We spent only 10 days in the montane forest and summit of Serra do Aracá due to difficulties accessing the site and remaining in it, and a limited time in the terra firme and

2014 Sociedade Brasileira de Zoologia | www.sbzoologia.org.br | www.scielo.br/zool All content of the journal, except where identified, is licensed under a Creative Commons attribution-type BY-NC. 
flooded forests. Therefore, sampling was more intense in white sand campinas and campinaranas (ANDERSON 1981), which are easy to access from rivers and are relatively low in species diversity.

The Aracá river valley and its tributaries are extensively covered with white sand campinas, sand dunes, low canopy forests (campinaranas) and black water flooded forests (igapó forests). In the Aracá valley, we also visited granite rock outcrops (inselbergs), with altitudes varying from 80-120 m and sparse vegetation cover. Hillside montane forests and the open vegetation of Serra do Aracá were found between 400 and 1,200 $\mathrm{m}$ a.s.l. Serra do Aracá has a flat table shape, which is typical of the Venezuelan tepuis (Huber 1988, Huber 2005) and its summit is occupied by partially flooded grasslands and scrublands and patches of low-canopy forests. The montane forests have a more heterogeneous vegetation structure and normally are covered by clouds in cold mornings. Detailed descriptions of the floristic and vegetation physiognomy of the Serra do Aracá can be found in Prance \& JoHnson (1992). Inventories of terra firme forests and igapó flooded forests were concentrated in Serra do Aracá foothills and along the lower courses of Aracá and Demini rivers (Fig. 1).

Our field methods included capture of birds with mist nets ( $36 \mathrm{~mm}$ mesh, $12 \times 2.5 \mathrm{~m}$ ), tape recording of bird vocalizations using professional tape recorders (Sony TCM 5000, Marantz PMD 660) and collection of specimens. We captured 635 individuals in 2,204 nets/hours of mist net operation. Biogeographic and/or taxonomic relevant specimens were collected (SISBIO \#32122-1) and deposited in the bird collection of Instituto Nacional de Pesquisas da Amazônia (INPA). Voice records will be deposited in public archives of the British Library's Sound Archive and the bird collection of the Instituto Nacional de Pesquisas da Amazônia (INPA). Meanwhile photographs or recordings can be requested from the authors (see Appendices S1-S6*).

Each species was recorded in the habitat categories defined in Appendix 1. Harrison indices were calculated between pairs of habitats using this categorization to determine the turnover of species between major habitats in the study region. This index is a measure of beta diversity, and is insensitive to differences in the total number of species of the sampling units compared (MAGURRAN 2004), which is useful for comparing habitats with very different numbers of species, as reported here. This index varies from 0 (total overlap in species composition) to 100 (completely different species composition) (Magurran 2004). Only species in the following habitats were included in the analysis: terra firme forests, tepuis (including montane forests plus grassland and scrub on the Serra do Aracá summit), igapó forests and white sand campinas and campinaranas.
We described the geographic distributions of individual bird species in the following categories: 1) monotypic or polytic species with widespread distribution in the Amazon, especially in the northern portion of the basin; 2) taxa (species or subspecies) whose distribution includes the left margin of the Rio Negro, including the Guianas and both margins of the Rio Branco. Some species in this category are also found in the southern part of the Amazon river, while others are not recorded in the Central Amazon; 3) taxa whose distribution falls primarily on the right margin of the Rio Negro, but which extends to the left margin (Negro/Branco interfluve). Some of these species can also be found in the south-western parts of the Amazon; 4) taxa apparently restricted to the north-western Amazon in the upper Rio Negro, South Venezuela, and Eastern Colombia; 5) taxa endemic to the Guyana Highlands (tepuis). Since no extensive collection of bird specimens has been conducted in the region, subspecies identification was tentative and made after extensive literature review (DEL Hoyo et al. 1992-2004, ResTALL et al. 2006), including regional catalogues (Friedmann 1948, Pinto 1966).

\section{RESULTS}

We compiled a check-list with 416 bird taxa, and documented the presence of 282 (68\%) of them (Appendix 1). The avifauna of Aracá is remarkable in the diversity of flycatchers (Tyrannidae: 36 species), antbirds (Thamnophilidae: 38 species), hummingbirds (Trochilidae: 25 species) and parrots (Psittacidae: 17 species). The list includes congeneric species that are not easy to tell appart in the field (Phaethornis superciliosus/malaris, Picumnus pumilus/lafresnayi), species that could be identified only to genus due inadequated documentation (Cypseloides sp., Topaza sp.) and some hypothetical records (Pyrrhura egregia (Sclater, 1881), Coccyzus melacoryphus Vieillot, 1817, Pteroglossus azara (Vieillot, 1819), Hylophilus thoracicus Temminck, 1822, Microcerculus ustulatus Salvin \& Godman, 1883) that neeed to be better documented. These records were maintained in the final check-list because they could be useful for further studies in the region.

During our fieldwork, we found bird species endemic to north-western Amazonia (e.g., Ammonastes pelzelni (Sclater, 1890), Cyanocorax heilprini Gentry, 1885), birds that are poorly known in nature (e.g., Crypturellus duidae Zimmer, 1938, Hemitriccus inornatus (Pelzeln, 1868)) or infrequently recorded for the Amazonia or Brazil (e.g., Sarkidiornis sylvicola Ihering \& Ihering, 1907, Leucophaeus atricilla (Linnaeus, 1758), Hydropsalis longirostris (Bonaparte, 1825), Campylopterus duidae Chapman, 1929). Further details of these records will be presented in another publication since the current study intends to describe the avifauna of the Aracá region in a more general context.

*Available as Online Supplementary Material accessed with the online version of the manuscript at http://www.scielo.br/zool 


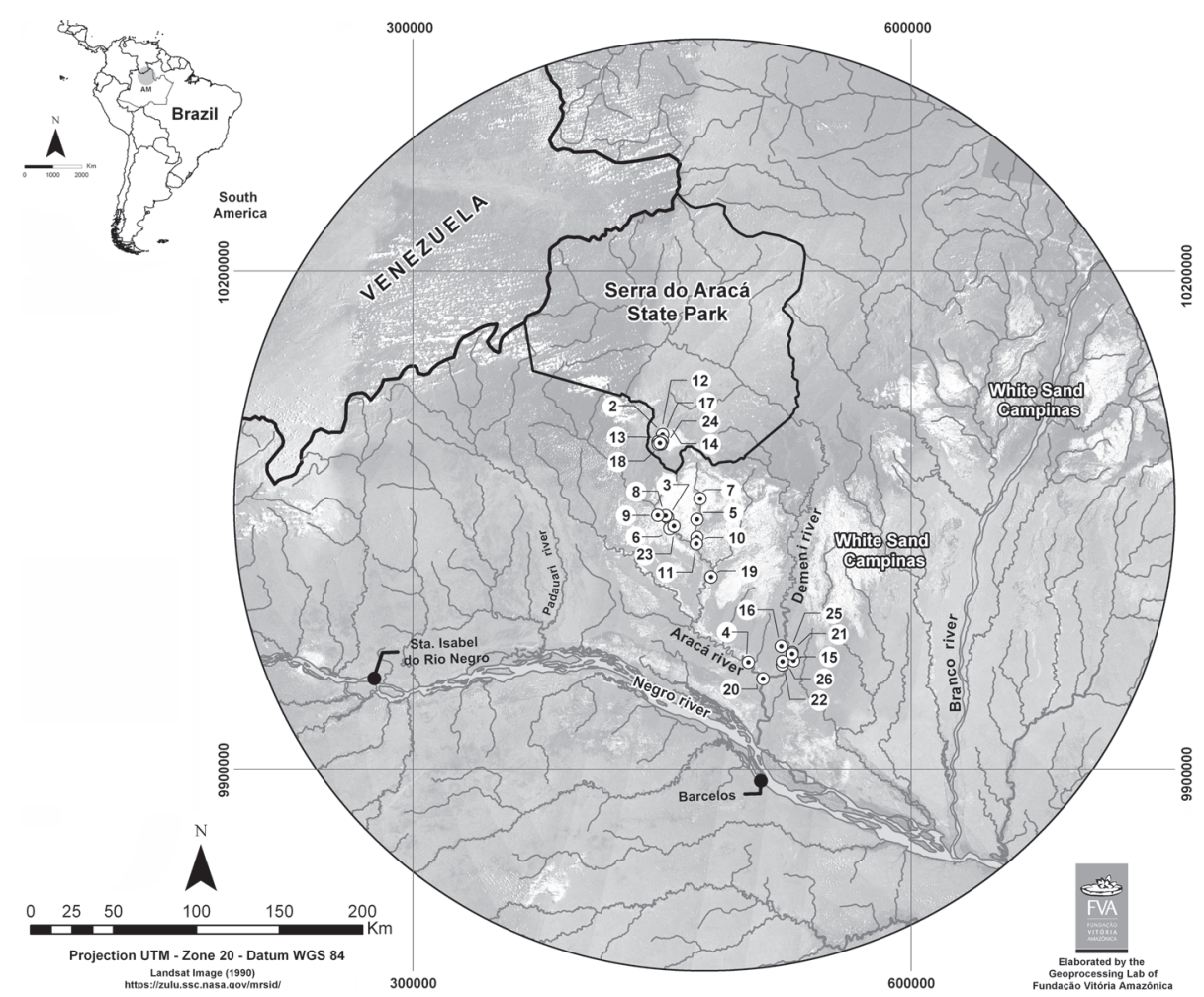

Figure 1. Northwestern region of the state of Amazonas showing the study sites, mainly rivers and municipalities. Note the large areas occupied by white sand campinas in the study region.

Generally, the diversity of birds in most habitats studied seems to be undersampled, considering the expected diversity found for similar habitats in more thoroughly studied regions (Table I). The exception was white sand campinas and campinaranas, where bird diversity was high compared with other better sampled regions (Table I). This result is likely related to the wide extension occupied by this habitat in Aracá (Fig. 1). Sampling efforts were less intense in terra firme forest and therefore it is likely that species diversity there is underestimated by at least $28 \%$ (Table I). Considering the deficit in the number of species found in the major habitats (Table I), we

Table I. Number of bird species recorded in the terrestrial habitats in the Aracá region compared with the same habitats in better sampled places in the Amazon.

\begin{tabular}{lccc}
\hline \multicolumn{1}{c}{ Habitats } & $\begin{array}{c}\text { Number of } \\
\text { recorded species }\end{array}$ & $\begin{array}{c}\text { Number of } \\
\text { expected species }\end{array}$ & $\begin{array}{c}\text { Aracá } \\
\text { deficits }\end{array}$ \\
\hline Terra firme forests & 179 & $250-2571$ & $71-78$ \\
Igapó flooded forests & 137 & $215^{2}$ & 78 \\
Tepuis & 36 & $14-47^{3}$ & 11 \\
White sand campinas & 180 & $153^{1}$ & - \\
and campinaranas & & & \\
\hline Sources: ${ }^{1}$ Borges \& AlmeIDA (2011), CoHN-Haft et al. (1997); ${ }^{2}$ Borges \& \\
Carvalmaes (2000), Borges \& AlmeldA (2011); ${ }^{3}$ Borges (unpubl. data).
\end{tabular}

expect that 160 to 167 species will be added to the current check-list in future surveys, increasing the known bird diversity of region to approximately 580 species.

A large proportion of birds (61\%, 231/379 species) were recorded in a single habitat. Most species were restricted to terra firme forest (27\%), followed by igapó forests (14\%), white sand campinas and campinaranas (16\%) and tepuis (4\%). Igapó flooded forests and white sand campinas and campinaranas have the most similar avifaunas (Table II). The avifauna of each vegetation type, however, is quite distinct in bird species composition, since Harrison index values were greater than 80 in most paired comparisons (Table II).

Table II. Harrison indices calculate in paired comparisons between major vegetation types in the Aracá region.

\begin{tabular}{lccc}
\hline & Igapó flooded forests & Tepuis & Terra firme forests \\
\hline $\begin{array}{l}\text { White sand campinas } \\
\text { and campinaranas }\end{array}$ & 57.1 & 83.3 & 69.3 \\
Igapó flooded forests & & & \\
Tepuis & 96.5 & 81.6 \\
\hline
\end{tabular}

Most avian taxa in the Aracá region are widely distributed throughout the Amazon basin (Table III). A smaller pro- 
Table III. Taxa (species or subspecies) recorded in Aracá region distributed in major biogeographic categories. For more details in categories definition see Methods section.

\begin{tabular}{lcl}
\hline Biogeographical categories & $\begin{array}{c}\text { Number of } \\
\text { species (\%) }\end{array}$ & Examples \\
\hline Taxa widely distributed in the Amazon & $200(67 \%)$ & Amazilia f. fimbriata Gmelin, 1788, Cacicus c. cela Linnaeus, 1766 \\
Taxa distributed mainly at left bank of the Rio Negro & $31(10 \%)$ & Todirostrum pictum Salvin, 1897, Monasa atra (Boddaert, 1783) \\
Taxa distributed mainly at right bank of the Rio Negro & $42(14 \%)$ & Hylophilus b. brunneiceps P.L. Sclater, 1866, Lepidothrix c. coronata carbonate Todd, 1925 \\
Taxa apparently restricted to Northwestern Amazonia & $12(4 \%)$ & Brotogeris chrysoptera tenuifrons Friedmann, 1945, Cyanocorax heilprini Gentry, 1885 \\
Taxa endemic to Guyana mountain system (tepuis) & $13(4 \%)$ & Trogon personatus Gould, 1842, Ceratopipra cornuta (Spix, 1825) \\
\hline
\end{tabular}

portion of representative taxa have their geographical distribution centered in the right or left banks of the Rio Negro, while only a few species have more restricted distributions (Table III)

\section{DISCUSSION}

Future inventories will significantly increase the bird diversity documented for the Aracá region. We recommend that additional inventories target the less known avifauna of the terra firme forests and tepuis. Also, increasing specimen collections is fundamental for understanding bird species diversity and biogeographical patterns in the region. Although incomplete, the results reported here suggests that large rivers (Branco and Negro), the Guyana mountain system and habitat diversity are the major determinants of the regional bird species distribution.

It is well-known that large rivers in the Amazon basin such as the Madeira, Negro and Solimões-Amazonas strongly influence the distribution of bird species (Hellmayr 1910, HafFer 1992, Borges 2007). In the study region, the Negro influences the geographic distribution of several bird species, especially in its lower course (BORGES 2007, NAKA 2011). Bird species such as Selenidera nattereri (Gould, 1836) and Tyranneutes stolzmanni (Hellmayr, 1906), recorded on the right margin of the Negro (Borges et al. 2001) and Aracá region, are substituted by related species (S. piperivora (Linnaeus, 1766), and T. virescens (Pelzeln, 1868)) on the left margin of Rio Branco near Manaus (CoHNHAFT et al. 1997). Additionally, some species typical of the left margin of Rio Branco (CoHN-HAFT et al. 1997), such as Monasa atra (Boddaert, 1783) and Gymnopithys rufigula, (Boddaert, 1783) were also recorded in the Aracá region and sites in southern Venezuela. These species are substituted by related species on the right margin of Rio Negro (COHN-HAFT et al. 1997, ZimMER \& Hilty 1997, Borges et al. 2001).

The avifauna of the Aracá region has a mixed composition, with species from both margins of the Rio Negro occurring sympatrically. Reinforcing the mixed nature of its avifauna, Aracá is located in a region of confluence of three areas of endemism for Amazonian birds: Imeri, Guianas and Pantepui (Cracraft 1985). The bird species C. heilprini and A. pelzelni are representatives of the Imeri area, while the Guiana is exempli- fied by the occurrence of Crax alector Linnaeus, 1766, M. atra and G. rufigula. The Pantepui area of endemism is characterized by birds of the Guyana highlands (MaYr \& Phelps 1967), and is represented in the study region by species that occur in Serra do Aracá, such as Ceratopipra cornuta (Spix, 1825) and Hylophilus sclateri Salvin \& Godman, 1883.

The patterns of species diversity and turnover of bird communities are clearly influenced by the various vegetation types found in the Aracá region, as shown by the high Harrison index values (Table II). The large difference in bird composition between habitats is partially explained by incomplete sampling, and it is likely that additional inventories will increase the similarity of bird species composition between them. However, natural history knowledge of several bird species suggests that the regional avifauna is well compartmentalized among the major regional landscapes.

These qualitative and preliminaries results suggests that the avifauna of the Aracá region is a product of interactive effects of geomorphological (development of rivers and mountains) and ecological (habitats diversity) processes. Further quantitative biogeographic analyses are needed to investigate the relative contribution of those processes in determining patterns of species distribution.

The extensive white sand campinas of the Aracá region, although relatively poor in species, contain large populations of birds that are specialized in this habitat type (Alonso \& Whitney 2003, Borges 2004, Aleixo \& Polleto 2007, Guilherme \& Borges 2011). These specialized birds are represented by subpopulations that are more or less isolated from each other through the Amazon basin, and hence this island-like habitat (Prance 1996) represents an ideal situation to evaluate the level of genetic and morphological divergence of birds and other organisms (see CAPURUCHO et al. 2013 for an illustrative example).

Serra Aracá is part of a mountain system with different levels of isolation that composes the biogeographical region of Pantepui (MAYr \& Phelps 1967). In Brazil, this biogeographical unit is represented by transnational (Brazil-Venezuela) mountains such as Pico da Neblina and Monte Roraima. Serra do Aracá is relatively isolated from a more continuous block of mountains and is entirely found in the Brazilian territory. Intensification of bird inventories in the Serra do Aracá will result in a better knowledge of the geographic distribution of 
the birds that are already known, and may result in the discovery of new taxa. A non-avian example of the potential of the Aracá region for taxonomic studies is the recent discovery of a new primate species of the genus Cacajao (Boubli et al. 2008).

The unique features of the Aracá region require conservation strategies that consider its biological and environmental diversity. Serra do Aracá State Park, with 1,818.700 ha, is one of the largest protected areas of the Brazilian Amazon. Unfortunately, the boundaries of this State Park do not include the large white sand campinas, the sand dunes and igapó flooded forests, which are found surrounding the Aracá river and its tributaries (Fig. 1). These habitats make an important contribution to the regional diversity of bird species. Moreover the occurrence of related taxa in the summits of the Serra do Aracá (e.g., Turdus ignobilis cf. murinus) and white sand campinas (e.g., Turdus ignobilis cf. arthuri) suggests that these birds share an evolutionary history (D.C. Oren unpubl. data). Also, the sand dunes of the Aracá region are relicts of unique geological events that occurred in the Holocene and Pleistocene (CARNeIro et al. 2002). Extending the boundaries of Serra do Aracá State Park or creating an adjacent newly protected area to incorporate the large extension of white sand campinas in the southern part of the Serra do Aracá would substantially improve the representation of the rich biological, historical and environmental heritage of the region.

\section{ACKNOWLEDGMENTS}

The field research in the Aracá region received financial support from the Gordon and Betty Moore Foundation through the Geopolitics of Conservation in the Rio Negro project. We would like to acknowledge the enthusiastic support of Enrique Ortiz and Jason Cole. Our fieldwork would not be possible without the collaboration of Zélio (Soldado), Raimundo, Roberto and Antenor. Carlos Durigan, Zig Kok and Marcelo Moreira who all shared important ornithological records. The State Center for Conservation Units (CEUC) and the Chico Mendes Institute for Biodiversity Conservation (ICMBIO) provided the formal licences for fieldwork and skin collections in the Serra do Aracá State Park. Thanks to our colleagues Carlos Durigan, Simone Iwanaga, Marcelo Moreira, Márcio Oliveira, Jansen Zuanon, Alberto Akama, Alexandre Loy (in memorian), Cintia Cornelius, Claudeir Vargas, Tiago, Célio, Daniel, Dalva and Cledson for the sense of humor during the hard work in the hills, sand fields, and rivers of the Aracá region. Cintia Cornelius kindly allowed us to include some of her species records in the check-list. In 2010, a field expediction to Aracá was supported by resources provided by Fundação de Amparo a Pesquisa do Amazonas (FAPEAM) and Fundação de Amparo a Pesquisa do Estado de São Paulo (FAPESP) through the FAPESP/FAPEAM joint funding program (09/53365-0). Marcelo Moreira kindly prepared the map. Nigel Smith, Yiyuan Jasmine and Karl Didier helped with the use of English language. Two anonymous reviewers helped to clarify important points in the text.

\section{LITERATURE CITED}

Aleixo, A. \& F. Poletto. 2007. Birds of an open vegetation enclave in southern Brazilian Amazonia. Wilson Journal of Ornithology 119: 610-630. doi: http://dx.doi.org/10. 1676/ 06-153.1

Alonso, J.A. \& B.M. Whitney. 2003. New distributional records of birds from white-sand forests of the northern Peruvian Amazon, with implications for biogeography of northern South America. Condor 105: 552-566. doi: http://dx.doi.org/ 10.1676/04-064

Anderson, A. 1981. White-sand vegetation of Brazilian Amazonia. Biotropica 13: 199-210. doi: http://dx.doi.org/ $10.2307 / 2388125$.

Ayres, M. \& T.H. Clutton-Brock. 1992. River boundaries and species range size in Amazonian primates. The American Naturalist 140: 531-537. doi: http://dx.doi.org/10.1086/ 285427.

BorgES, S.H. 2004. Species poor but distinct: bird assemblages in white sand vegetation in Jaú National Park, Amazonian Brazil. Ibis 146: 114-124. doi: 10.1111/j.1474-919X.2004.00230.x

Borges, S.H. 2007. Análise biogeográfica da avifauna da região oeste do baixo Rio Negro, Amazônia Brasileira. Revista Brasileira de Zoologia 24: 919-940. doi: http://dx.doi.org/ 10.1590/S0101-81752007000400008

Borges, S.H. \& A.M.P. Carvalhaes. 2000. Bird species of black water inundation forest in the Jaú National Park (Amazonas State, Brazil): their contribution to regional species richness. Biodiversity and Conservation 9: 201-214. doi: 10.1023/A:1008902306499.

Borges, S.H. \& R.A. Almeida. 2011. Birds of the Jaú National Park and adjacent area, Brazilian Amazon: new species records with reanalysis of a previous checklist. Revista Brasileira de Ornitologia 19: 108-133.

Borges, S.H. \& J.M.C. Silva. 2012. A new area of endemism for Amazonian birds in the Rio Negro basin. Wilson Journal of Ornithology 124: 15-24. doi: http://dx.doi.org/10.1676/ 07-103.1

Borges, S.H.; M. Cohn-Haft; A.M.P. Carvalhaes; L.M. Henriques; J.F. Pacheco \& A. Whittaker. 2001. Birds of Jaú National Park, Brazilian Amazon: Species check-list, biogeography and conservation. Ornitologia Neotropical 12: 109-140.

Boubli, J.P.; M.N.F. Silva; M.V. Amado; T. Hrbek; F.B. Pontual \& I.P. FARIAS. 2008. A Taxonomic Reassessment of Cacajao melanocephalus Humboldt (1811), with the description of two new species. International Journal of Primatology 29: 723-741.

Capurucho, J.C.; C. Cornelius, S.H. Borges; M. Cohn-Haft; A. Aleixo; J.P. Metzger \& C. Ribas. 2013. Combining phylogeography and landscape genetics of Xenopipo atronitens (Aves: Pipridae), a white sand campinas specialist, to understand Pleistocene landscape evolution in Amazonia. Biological Journal of the Linnean Society 110: 60-76. doi: 10.1111/bij.12102 
Carneiro Filho, A.C.; D. Schwartz; S.H. Tatumi \& T. Rosique. 2002. Amazonian paleodunes provide evidence for a drier climate phases during the late Pleistocene-Holocene. Quaternary Research 58: 205-209. doi:10.1006/qres.2002.2345

CBRO. 2014. Listas das aves do Brasil. Comitê Brasileiro de Registros Ornitológicos, avaliable online at: http:// www.cbro.org.br. [Accessed: May 2014].

Cohn-Haft, M.; A.M.F. Pacheco; C. Bechtoldt; M.F. Torres; A.M. Fernandes; C.H. SARdelli; I.T. Macedo. 2007a. Inventário ornitológico, p. 145-178. In: L. Rapp Py-Daniel; C.P. Deus; A.L. Henriques; D.M. Pimpão; O.M. Ribeiro (Orgs). Biodiversidade do Médio Madeira: Bases científicas para propostas de conservação. Manaus, INPA, 244p.

Cohn-Haft, M.; A. Whittaker \& P.C. Stouffer. 1997. A new look at the "species-poor" Central Amazon: The avifauna north of Manaus, Brazil. Ornithological Monographs 48: 205235.

Cohn-Haft, M; L.N. Naka \& A.M. Fernandes. 2007b. Padrões de distribuição da avifauna da várzea dos rios Solimões e Amazonas, p. 287-323. In: A.L. Albernaz (Org.). Conservação da várzea: identificação e caracterização de regiões biogeográficas. Manaus, Ibama/ProVárzea, 353p.

Cracraft, J. 1985. Historical biogeography and patterns of differentiation within the South American avifauna: Areas of endemisms. Ornithological Monographs 36: 49-84.

Del Hoyo, J.; A. Elliot \& J. Sargatal. 1992-2004. Handbook of the Birds of the World. Barcelona, Linx Edicions, 1992: vol. 1 - Ostrich to Ducks; 1993: vol. 2 - New World Vultures to Guineafowl; 1996: vol. 3 Hoatzin to Auks; 1997: vol. 4 Sandgrouse to Cuckoos; 1999: vol. 5 - Barn-Owls to Hummingbirds; 2001: vol. 6 - Mousebirds to Hornbills; 2002: vol. 7 - Jacamars to Woodpeckers; 2003: vol. 8 Broadbills to Tapaculos; 2004: vol. 9 - Cotingas to Wagtails.

FriedmanN, H. 1948. Birds collected by the National Geographic Society's expeditions to northern Brazil and southern Venezuela. Proceedings of the United States National Museum 97: 373-569.

Guilherme, E. \& S.H. Borges. 2011. Ornithological records from a campina/campinarana enclave on the upper Juruá river, Acre, Brazil. Wilson Journal of Ornithology 123: 24-32. doi: http://dx.doi.org/10.1676/10-036.1

Haffer, J. 1969. Speciation in Amazonian birds. Science 165: 131-137. doi: 10.1126/science.165.3889.131

Haffer, J. 1974. Avian speciation in tropical South America. Publications of the Nuttall Ornithological Club 14: 1-390.

Haffer J. 1992. On the "river effect" in some forest birds of southern Amazonia. Boletim do Museu Paraense Emílio Goeldi, Série Zoologia, 8: 217-245.

Hellmayr, C.E. 1910. The birds of the rio Madeira. Novitates Zoologicae 17: 257-428.

Huber, O. 1988. Vegetacion y flora de Pantepui, Region Guayana. Acta Botanica Brasilica 1 (2): 41-52. http:// dx.doi.org/10.1590/S0102-33061987000300005
Huber, O. 2005. Diversity of vegetation types in the Guyana Region: an overview. Biologiske Skrifter 55: 169-188.

MagurRan, A. 2004. Measuring biological diversity. London, Blackwell Publishing.

MaYr, E. \& W.H. Phelps. 1967. The origin of the bird fauna of the South Venezuelan highlands. Bulletin of the American Museum of Natural History 136: 273-327.

NAKA, L.N. 2011. Avian distribution patterns in the Guiana Shield: implications for the delimitation of Amazonian areas of endemism. Journal of Biogeography 38: 681-696. doi: 10.1111/j.1365-2699.2010.02443.x

NaKa, L.N.; M. Cohn-Haft; F. Mallet-Rodrigues; M.P.D. Santos \& M.F. Torres. 2006. The avifauna of the Brazilian state of Roraima: Bird distribution and biogeography in the Rio Branco basin. Revista Brasileira de Ornitologia 14: 197-238.

NAKA, L.N.; M. Cohn-Haft; A. WhitTaKer; J.M. BarnetT \& M.F. ToRRES. 2007. Avian biogeography of amazonian flooded forests in the Rio Branco basin, Brazil. Wilson Journal of Ornithology 119: 439-449. doi: http://dx.doi.org/10.1676/06-062.1

Oren, D.C. 2001. Biogeografia e conservação de aves na região Amazônica, p. 97-109. In: J.P. СAPOBIANCO (Orgs). Biodiversidade da Amazônia Brasileira. São Paulo, Estação Liberdade: Instituto Socioambiental.

Oren, D.C. \& H.G. Albuquerque. 1991. Priority areas for new avian collections in Brazilian Amazonia. Goeldiana, Zoologia, 6: 1-11.

PINTO, O. 1966. Estudo crítico e catálogo remissivo das aves do território federal de Roraima. Cadernos da Amazônia 8: 1-176.

PranCE, G. 1996. Islands in Amazonia. Philosophical Transactions of the Royal Society B 351: 823-833.

Prance, G.T. \& D.M. Johnson. 1992. Plant collections from the plateau of Serra do Aracá (Amazonas, Brazil) and their phytogeographic affinities. Kew Bulletin 47: 1-22.

Rahbeк, C. \& G. Graves. 2001. Multiscale assessment of patterns of avian species richness. Proceedings of the National Academy of Sciences of the United States of America 98: 4534-4539. doi: 10.1073/pnas.071034898

Restall, R.; C. Rodner \& M. Lentino. 2006. Birds of Northern South America - an identification guide. London, Helm Field Guides, Christopher Helm.

Santos, J.O.S.; B.W. Nelson \& A. Giovaninni. 1993. Dunas gigantes e campos de areia. Ciência Hoje 93: 22-25.

Santos, M.P.D. \& A. Aleixo. 2011. Foreword. Revista Brasileira de Ornitologia 19 (2): 1.

Silva, J.M.C.; A.B. Rylands \& G.A.B. Fonseca. 2005. The fate of the Amazonian areas of endemism. Conservation Biology 19: 689-694. doi: 10.1111/j.1523-1739.2005.00705.x

ZimMER, K. \& S. Hilty. 1997. Avifauna of a locality in the upper Orinoco drainage of Amazonas, Venezuela. Ornithological Monographs 48: 865-886.

Submitted: 07.II.2013; Accepted: 04.V.2014. Editorial responsibility: Fernando de C. Passos 
Appendix 1. List of bird species recorded in the Aracá region, Amazonas State, Brazilian Amazon. Taxonomic nomenclature follows the Brazilian Committee of Ornithological Records (CBRO 2014). Species between parentheses do not have adequate documentation for a safe identification.

\begin{tabular}{|c|c|c|c|c|c|}
\hline Taxons & Portuguese common name & Evidences $^{1}$ & Habitats $^{2}$ & Sites $^{3}$ & Sources ${ }^{4}$ \\
\hline \multicolumn{6}{|l|}{ Tinamidae Gray, 1840} \\
\hline Tinamus major (Gmelin, 1789) & inhambu-de-cabeça-vermelha & $g(2), v(1)$ & $\mathrm{mtf}$ & $1,4,15$ & $a w, s r$ \\
\hline Crypturellus cinereus (Gmelin, 1789) & inhambu-preto & $v(2)$ & $\mathrm{ca}$ & 9 & sc \\
\hline Crypturellus soui (Hermann, 1783) & tururim & $g(1,2)$ & $\mathrm{cp}, \mathrm{mtf}, \mathrm{ca}$ & $1,4,6,10,22$ & aw, s2, sr \\
\hline Crypturellus undulatus (Temminck, 1815) & jaó & $g(1,2)$ & ig, $\mathrm{cp}$ & 4 & $a w, s r$ \\
\hline Crypturellus duidae Zimmer, 1938 & inhambu-de-pé-cinza & $g(1,2)$ & $\mathrm{ca}, \mathrm{mtf}$ & $4,21,24$ & aw, sr \\
\hline \multicolumn{6}{|l|}{ Anatidae Leach, 1820} \\
\hline Dendrocygna autumnalis (Linnaeus, 1758) & asa-branca & $\vee(1)$ & $\mathrm{r}$ & 4 & aw \\
\hline Cairina moschata (Linnaeus, 1758) & pato-do-mato & $o(1,2,3)$ & $\mathrm{r}, \mathrm{ca}$ & $1,4,20$ & $a w, s 2, s r$ \\
\hline Sarkidiornis sylvicola Ihering \& Ihering, 1907 & pato-de-crista & $\circ(1)$ & $r$ & 4 & aw \\
\hline \multicolumn{6}{|l|}{ Cracidae Rafinesque, 1815} \\
\hline Ortalis motmot (Linnaeus, 1766) & aracuã-pequeno & $g(1,2)$ & $\mathrm{cp}, \mathrm{mm}, \mathrm{ca}$ & $4,5,8,9$ & aw, s2 \\
\hline Penelope jacquacu Spix, 1825 & jacu-de-spix & $g(1), f(3), \circ(2), v(2)$ & $\mathrm{mtf}$ & $1,4,12,16-18,22,26$ & $a w, s 2, s r$ \\
\hline Pauxi tomentosa (Spix, 1825) & mutum-do-norte & $g(1,2), f(2), o(2)$ & $\mathrm{ca}$ & 4 & $a w, s 2, s r$ \\
\hline Crax alector Linnaeus, 1766 & mutum-poranga & $g(2), f(4 a), v(1)$ & $\mathrm{mtf}$ & $4,16,21$ & aw, s2, sr, ter \\
\hline \multicolumn{6}{|l|}{ Odontophoridae Gould, 1844} \\
\hline Odontophorus gujanensis (Gmelin, 1789) & uru-corcovado & $\circ(2)$ & $\mathrm{mtf}$ & $1,17,21,25$ & $\mathrm{sr}$ \\
\hline \multicolumn{6}{|l|}{ Ciconiidae Sundevall, 1836} \\
\hline Ciconia maguari (Gmelin, 1789) & maguari & $o(2)$ & r, ca & 11 & s2 \\
\hline Jabiru mycteria (Lichtenstein, 1819) & tuiuiú & $o(1)$ & $r$ & 4 & aw \\
\hline Mycteria americana Linnaeus, 1758 & cabeça-seca & $\circ(1,2)$ & $r$ & 4 & aw, s2 \\
\hline \multicolumn{6}{|l|}{ Phalacrocoracidae Reichenbach, 1849} \\
\hline Phalacrocorax brasilianus (Gmelin, 1789) & biguá & $\circ(2)$ & $r$ & 1 & s2 \\
\hline \multicolumn{6}{|l|}{ Anhingidae Reichenbach, 1849} \\
\hline Anhinga anhinga (Linnaeus, 1766) & biguatinga & $\circ(1,2,3)$ & $\mathrm{r}$ & $1,4,20$ & $a w, s 2, s r$ \\
\hline \multicolumn{6}{|l|}{ Ardeidae Leach, 1820} \\
\hline Tigrisoma lineatum (Boddaert, 1783) & socó-boi & $\circ(1,2)$ & $\mathrm{r}, \mathrm{ca}$ & 4 & aw, s2 \\
\hline Cochlearius cochlearius (Linnaeus, 1766) & arapapá & $\vee(1)$ & $r$ & 4 & aw \\
\hline Butorides striata (Linnaeus, 1758) & socozinho & $g(2), \circ(1,2)$ & $\mathrm{r}$ & $1,4,20$ & $a w, s 2, s r$ \\
\hline Bubulcus ibis (Linnaeus, 1758) & garça-vaqueira & $\circ(1,2)$ & $r$ & 4,20 & $a w, s r$ \\
\hline Ardea cocoi Linnaeus, 1766 & garça-moura & $\circ(1,2)$ & $r$ & 1,4 & $a w, s r$ \\
\hline Ardea alba Linnaeus, 1758 & garça-branca-grande & $\circ(1,2,3)$ & $\mathrm{r}, \mathrm{ca}$ & $1,4,8,20$ & $a w, s 2, s r$ \\
\hline Pilherodius pileatus (Boddaert, 1783) & garça-real & $\circ(1,2,3)$ & $r$ & 4 & aw, s2 \\
\hline Egretta thula (Molina, 1782) & garça-branca-pequena & $\circ(1,2,3)$ & $\mathrm{r}$ & 1,4 & $a w, s 2, s r$ \\
\hline \multicolumn{6}{|l|}{ Threskiornithidae Poche, 1904} \\
\hline Mesembrinibis cayennensis (Gmelin, 1789) & coró-coró & $g(2), \circ(1,2)$ & $\mathrm{r}, \mathrm{ca}$ & 1,4 & $a w, s 2, s r$ \\
\hline \multicolumn{6}{|l|}{ Cathartidae Lafresnaye, 1839} \\
\hline Cathartes aura (Linnaeus, 1758) & urubu-de-cabeça-vermelha & $\circ(1,2)$ & $\mathrm{ca}, \mathrm{ig}, \mathrm{mtf}, \mathrm{cp}$ & $2,4,8$ & $a w, s 2, s r$ \\
\hline Cathartes melambrotus Wetmore, 1964 & urubu-da-mata & $\circ(1,2)$ & $\mathrm{ca}, \mathrm{ig}, \mathrm{mtf}, \mathrm{cp}$, cal & 4 & aw, s2 \\
\hline Coragyps atratus (Bechstein, 1793) & urubu-de-cabeça-preta & $\circ(1,2)$ & $\mathrm{ca}, \mathrm{ig}, \mathrm{mtf}, \mathrm{cp}$ & 1,4 & $a w, s 2, s r$ \\
\hline Sarcoramphus papa (Linnaeus, 1758) & urubu-rei & $\circ(2)$ & $\mathrm{mtf}$, ig & 1,15 & $s 2, s r$ \\
\hline \multicolumn{6}{|l|}{ Pandionidae Bonaparte, 1854} \\
\hline Pandion haliaetus (Linnaeus, 1758) & águia-pescadora & $\circ(1,2)$ & ig & $1,4,20$ & $a w, s r$ \\
\hline
\end{tabular}


Appendix 1. Continued.

\begin{tabular}{|c|c|c|c|c|c|}
\hline Taxons & Portuguese common name & Evidences $^{1}$ & Habitats $^{2}$ & Sites $^{3}$ & Sources ${ }^{4}$ \\
\hline \multicolumn{6}{|l|}{ Accipitridae Vigors, 1824} \\
\hline Elanoides forficatus (Linnaeus, 1758) & gavião-tesoura & $\circ(2)$ & ca, ig & 1 & s2, sr \\
\hline Ictinia plumbea (Gmelin, 1788) & sovi & $\circ(1)$ & ig & 4 & aw, s2 \\
\hline Accipiter superciliosus (Linnaeus, 1766) & gavião-miudinho & $\circ(1)$ & ig & 4,20 & aw \\
\hline Geranospiza caerulescens (Vieillot, 1817) & gavião-pernilongo & $\circ(2)$ & ig & 20 & sr \\
\hline Leucopternis melanops (Latham, 1790) & gavião-de-cara-preta & $v(3)$ & $\mathrm{mtf}$ & 1 & sr \\
\hline Urubitinga urubitinga (Gmelin, 1788) & gavião-preto & $\circ(1,2)$ & ig & $1,4,20$ & $a w, s r$ \\
\hline Heterospizias meridionalis (Latham, 1790) & gavião-caboclo & $\circ(2)$ & ca & 9 & sc \\
\hline Busarellus nigricollis (Latham, 1790) & gavião-belo & $o(1,2)$ & ig & 4,20 & aw, sr \\
\hline Rupornis magnirostris (Gmelin, 1788) & gavião-carijó & $g(1,2), f(2)$ & ig, ca, cp & $\begin{array}{l}1,4-6,8,10,20,- \\
26\end{array}$ & $a w, s 2, s r$ \\
\hline Spizaetus tyrannus (Wied, 1820) & gavião-pega-macaco & $o(4 a), v(2)$ & $\mathrm{mtf}$ & 22 & s2, ter \\
\hline Spizaetus ornatus (Daudin, 1800) & gavião-de-penacho & $\circ(4 a), v(2)$ & $\mathrm{mtf}$ & 22 & s2, sr, ter \\
\hline \multicolumn{6}{|l|}{ Eurypygidae Selby, 1840} \\
\hline Eurypyga helias (Pallas, 1781) & pavãozinho-do-pará & $\circ(1,2)$ & $r$ & 1,4 & aw, sr \\
\hline \multicolumn{6}{|l|}{ Aramidae Bonaparte, 1852} \\
\hline Aramus guarauna (Linnaeus, 1766) & carão & $o(1,2)$ & $r$ & 3,27 & $a w, s 2$ \\
\hline \multicolumn{6}{|l|}{ Psophiidae Bonaparte, 1831} \\
\hline Psophia crepitans Linnaeus, 1758 & jacamim-de-costas-cinzentas & $f(2,4 a)$ & $\mathrm{mtf}$ & 1,16 & $s 1, s 2, s r$ \\
\hline \multicolumn{6}{|l|}{ Rallidae Rafinesque, 1815} \\
\hline Aramides cajaneus (Statius Muller, 1776) & saracura-três-potes & $\vee(2)$ & $\mathrm{ca}$ & 19,20 & s2, sr \\
\hline Laterallus viridis (Statius Muller, 1776) & sanã-castanha & $g(2)$ & $\mathrm{ca}$ & 8 & s2 \\
\hline \multicolumn{6}{|l|}{ Heliornithidae Gray, 1840} \\
\hline Heliornis fulica (Boddaert, 1783) & picaparra & $\circ(2)$ & $r$ & 1 & s2 \\
\hline \multicolumn{6}{|l|}{ Charadriidae Leach, 1820} \\
\hline Vanellus cayanus (Latham, 1790) & batuíra-de-esporão & $f(4-b), o(1,2)$ & $r$ & 1,4 & $a w, s 1, s 2, s r$, ter \\
\hline Vanellus chilensis (Molina, 1782) & quero-quero & $g(2), \circ(1,2,3)$ & $\mathrm{ca}, \mathrm{r}$ & $1,4-6,8,10,11,19$ & aw, s2, sr \\
\hline Charadrius collaris Vieillot, 1818 & batuíra-de-coleira & $\circ(1)$ & $\mathrm{r}, \mathrm{ca}$ & 4 & aw \\
\hline \multicolumn{6}{|l|}{ Scolopacidae Rafinesque, 1815} \\
\hline Gallinago paraguaiae (Vieillot, 1816) & narceja & $\begin{array}{l}\text { g (1), o (2), c (4d) } \\
\text { - INPA } 2077\end{array}$ & $\mathrm{~mm}, \mathrm{ca}$ & 4 & $a w, s 2$ \\
\hline Actitis macularius (Linnaeus, 1766) & maçarico-pintado & $\circ(1,3)$ & $r$ & 1,4 & $a w, s r$ \\
\hline Tringa solitaria Wilson, 1813 & maçarico-solitário & $o(1,3)$ & $r$ & 1,4 & aw, sr \\
\hline Tringa melanoleuca (Gmelin, 1789) & maçarico-grande-de-perna-amarela & $\circ(1)$ & $r$ & 4 & aw \\
\hline Tringa flavipes (Gmelin, 1789) & maçarico-de-perna-amarela & $\circ(1)$ & $r$ & 4 & aw \\
\hline \multicolumn{6}{|l|}{ Jacanidae Chenu \& Des Murs, 1854} \\
\hline Jacana jacana (Linnaeus, 1766) & jaçanã & $\circ(1)$ & $r$ & 4 & aw \\
\hline \multicolumn{6}{|l|}{ Laridae Rafinesque, 1815} \\
\hline Leucophaeus atricilla (Linnaeus, 1758) & gaivota-alegre & $o(1)$ & $r$ & 4 & aw \\
\hline \multicolumn{6}{|l|}{ Sternidae Vigors, 1825} \\
\hline Sternula superciliaris (Vieillot, 1819) & trinta-réis-anão & $\circ(1)$ & $r$ & 4 & aw \\
\hline Phaetusa simplex (Gmelin, 1789) & trinta-réis-grande & $g(1)$ & $r$ & 1,4 & $a w, s 2, s r$ \\
\hline \multicolumn{6}{|l|}{ Rynchopidae Bonaparte, 1838} \\
\hline Rynchops niger Linnaeus, 1758 & talha-mar & $g(1)$ & $r$ & 4 & aw \\
\hline
\end{tabular}

Columbidae Leach, 1820 
Appendix 1. Continued.

\begin{tabular}{|c|c|c|c|c|c|}
\hline Taxons & Portuguese common name & Evidences $^{1}$ & Habitats $^{2}$ & Sites $^{3}$ & Sources $^{4}$ \\
\hline Columbina passerina (Linnaeus, 1758) & rolinha-cinzenta & $g(1), f(2)$ & $\mathrm{ca}, \mathrm{cp}$ & $4,19,20,23$ & $a w, s 2, s r$ \\
\hline Patagioenas speciosa (Gmelin, 1789) & pomba-trocal & $g(2), v(2)$ & $\mathrm{ca}$, ig & 24 & s2, sr \\
\hline Patagioenas fasciata (Say, 1823) & pomba-de-coleira-branca & $\circ(3)$ & cal & 2 & $\mathrm{sr}$ \\
\hline Patagioenas cayennensis (Bonnaterre, 1792) & pomba-galega & $g(1,2), o(2), v(2)$ & ig, cp, ca & $1,4-11,19,20$ & aw, s2, sr \\
\hline Patagioenas plumbea (Vieillot, 1818) & pomba-amargosa & $g(1,2)$ & $\mathrm{mtf}, \mathrm{ig}, \mathrm{ca}$ & $4,16,20-22,25$ & $a w, s r$ \\
\hline Patagioenas subvinacea (Lawrence, 1868) & pomba-botafogo & $g(1,2)$ & $\mathrm{mtf}$ & $4,12,17,18,21$ & $a w, s r$ \\
\hline Leptotila verreauxi Bonaparte, 1855 & juriti-pupu & $g(2)$ & $\mathrm{ca}, \mathrm{mtf}$ & 24 & s2, sr \\
\hline Leptotila rufaxilla (Richard \& Bernard, 1792) & juriti-gemedeira & $g(1,2)$ & $\mathrm{ca}$, ig & $4,18,20,22$ & $a w, s r$ \\
\hline Geotrygon montana (Linnaeus, 1758) & pariri & $g(2)$ & ca & $15-18,21,22,24$ & $\mathrm{sr}$ \\
\hline \multicolumn{6}{|l|}{ Opisthocomidae Swainson, 1837} \\
\hline Opisthocomus hoazin (Statius Muller, 1776) & cigana & $g(2), f(2), o(1,2,3)$ & ig & $1,4,20$ & aw, sr \\
\hline \multicolumn{6}{|l|}{ Cuculidae Leach, 1820} \\
\hline Coccycua minuta (Vieillot, 1817) & chincoã-pequeno & $\circ(2,3)$ & ig & 20 & $\mathrm{sr}$ \\
\hline Piaya cayana (Linnaeus, 1766) & alma-de-gato & $g(2), o(1,2)$ & ig, ca, cp & $4,8,20$ & aw, s2, sr \\
\hline Piaya melanogaster (Vieillot, 1817) & chincoã-de-bico-vermelho & $\circ(2)$ & $\mathrm{mtf}$ & $17,18,22,25$ & $\mathrm{sr}$ \\
\hline Coccyzus melacoryphus Vieillot, 1817 & papa-lagarta-acanelado & $o(2,3)$ & ig, ca & 20 & $\mathrm{sr}$ \\
\hline Crotophaga major Gmelin, 1788 & anu-coroca & $g(1), v(2)$ & ig & $1,4,20$ & $a w, s 2, s r$ \\
\hline Crotophaga ani Linnaeus, 1758 & anu-preto & $\circ(2,3)$ & ig, $\mathrm{cp}$ & 20 & $\mathrm{~s} 2, \mathrm{sr}$ \\
\hline Tapera naevia (Linnaeus, 1766) & saci & $\vee(1)$ & $\mathrm{ca}$ & 4 & aw \\
\hline Neomorphus rufipennis (Gray, 1849) & jacu-estalo-de-asa-vermelha & o (4a) & $\mathrm{mtf}$ & 18 & s2, ter \\
\hline \multicolumn{6}{|l|}{ Strigidae Leach, 1820} \\
\hline Megascops choliba (Vieillot, 1817) & corujinha-do-mato & $v(1,2)$ & $\mathrm{ca}$, ig & 4,9 & aw, s2 \\
\hline Megascops watsonii (Cassin, 1849) & corujinha-orelhuda & $v(1), f(2)$ & $\mathrm{mtf}$ & $4,15,18$ & $a w, s r$ \\
\hline Pulsatrix perspicillata (Latham, 1790) & murucututu & $\vee(1)$ & ig & 4 & aw \\
\hline Athene cunicularia (Molina, 1782) & coruja-buraqueira & $f(2)$ & $\mathrm{ca}$ & 19 & s2 \\
\hline \multicolumn{6}{|l|}{ Nyctibiidae Chenu \& Des Murs, 1851} \\
\hline Nyctibius griseus (Gmelin, 1789) & mãe-da-lua & $g(2), v(1)$ & $\mathrm{ca}$, ig & $1,4,5,10,11,15,24$ & aw, s2, sr \\
\hline \multicolumn{6}{|l|}{ Caprimulgidae Vigors, 1825} \\
\hline Lurocalis semitorquatus (Gmelin, 1789) & tuju & $\vee(1)$ & $\mathrm{mtf}$ & 4 & aw \\
\hline Chordeiles pusillus Gould, 1861 & bacurauzinho & $g(1,2)$ & $\mathrm{ca}$ & $4,5,6,8-11,19$ & aw, s2 \\
\hline Hydropsalis leucopyga (Spix, 1825) & bacurau-de-cauda-barrada & $g(1), v(2)$ & $\mathrm{ca}$, ig & $1,4,8,20$ & aw, s2, sr \\
\hline Hydropsalis albicollis (Gmelin, 1789) & bacurau & $g(2), v(1,2)$ & $\mathrm{ca}$, ig & $1,4-6,8,10,11,15,19$ & aw, s2, sr \\
\hline Antrostomus rufus (Boddaert, 1783) & joão-corta-pau & $g(1,2)$ & $\mathrm{ca}$ & $4,6,9,24$ & aw, s2, sr \\
\hline Hydropsalis longirostris (Bonaparte, 1825) & bacurau-da-telha & $f(3)$ & cal & 2 & $\mathrm{sr}$ \\
\hline Hydropsalis cayennensis (Gmelin, 1789) & bacurau-de-cauda-branca & $g(2)$ & $\mathrm{ca}$ & $2,5,6,9-11,19$ & s2, sr \\
\hline Hydropsalis nigrescens (Cabanis, 1848) & bacurau-de-lajeado & $g(2), o(1)$ & $\mathrm{ca}$ & 4,24 & $a w, s r$ \\
\hline Hydropsalis climacocerca (Tschudi, 1844) & acurana & $g(1)$ & ig & 4,20 & $a w, s r$ \\
\hline \multicolumn{6}{|l|}{ Apodidae Olphe-Galliard, 1887} \\
\hline Cypseloides sp. & taperuçu-velho & $\circ(3)$ & $\mathrm{cal}$ & 2 & $\mathrm{sr}$ \\
\hline Streptoprocne zonaris (Shaw, 1796) & taperuçu-de-coleira-branca & $\circ(2,3)$ & $\mathrm{cal}, \mathrm{ca}$ & 2 & $\mathrm{~s} 2, \mathrm{sr}$ \\
\hline Chaetura spinicaudus (Temminck, 1839) & andorinhão-de-sobre-branco & $g(1)$ & $\mathrm{ca}$, ig & 4 & aw \\
\hline Chaetura brachyura (Jardine, 1846) & andorinhão-de-rabo-curto & $\circ(1)$ & $\mathrm{ca}$, ig & 4 & aw \\
\hline $\begin{array}{l}\text { Aeronautes montivagus (d'Orbigny \& } \\
\text { Lafresnaye, 1837) }\end{array}$ & andorinhão-serrano & $\circ(2)$ & $\mathrm{ca}$, ig & $2,6,24$ & $\mathrm{~s} 2, \mathrm{sr}$ \\
\hline & & & & & Cont \\
\hline
\end{tabular}


Appendix 1. Continued.

\begin{tabular}{|c|c|c|c|c|c|}
\hline Taxons & Portuguese common name & Evidences $^{1}$ & Habitats $^{2}$ & Sites $^{3}$ & Sources $^{4}$ \\
\hline \multicolumn{6}{|l|}{ Trochilidae Vigors, 1825} \\
\hline Threnetes leucurus (Linnaeus, 1766) & balança-rabo-de-garganta-preta & $f(2)$ & $\mathrm{mtf}, \mathrm{cp}$ & $12,13,17,18,22,24$ & $\mathrm{sr}$ \\
\hline Phaethornis rupurumii Boucard, 1892 & rabo-branco-do-rupununi & $g(2), f(2), v(1)$ & $\mathrm{ca}$ & 4 & aw, s1 \\
\hline Phaethornis ruber (Linnaeus, 1758) & rabo-branco-rubro & $g(1,2), f(2)$ & $\mathrm{mtf}, \mathrm{cp}, \mathrm{ca}$ & $4,12,15-18,21,22,24$ & $a w, s 1, s r$ \\
\hline Phaethornis bourcieri (Lesson, 1832) & rabo-branco-de-bico-reto & $\circ(2,3)$ & $\mathrm{mtf}$ & 13,17 & $\mathrm{sr}$ \\
\hline $\begin{array}{l}\text { Phaethornis superciliosus (Linnaeus, 1766)/P. } \\
\text { malaris (Nordmann, 1835) }\end{array}$ & rabo-branco-de-bigodes & $g(2), v(1)$ & $\mathrm{mtf}$ & $4,12,14,16-18,20,22$ & $a w, s r$ \\
\hline Doryfera johannae (Bourcier, 1847) & bico-de-lança & $g(2), f(2)$ & cal & 2 & s1, s2 \\
\hline Campylopterus largipennis (Boddaert, 1783) & asa-de-sabre-cinza & $\circ(2,3)$ & $\mathrm{mtf}$ & 13,17 & $\mathrm{sr}$ \\
\hline Campylopterus duidae Chapman, 1929 & asa-de-sabre-de-peito-camurça & $f(2)$ & cal & 2 & s2 \\
\hline Florisuga mellivora (Linnaeus, 1758) & beija-flor-azul-de-rabo-branco & $\circ(2)$ & $\mathrm{mtf}$ & 20 & $\mathrm{sr}$ \\
\hline Colibri delphinae (Lesson, 1839) & beija-flor-marrom & $\begin{array}{l}g(2), f(2), c(2)- \\
\text { INPA } 2432\end{array}$ & cal & 2 & s2 \\
\hline Topaza sp. & beija-flor-brilho-de-fogo & $g(2)$ & $\mathrm{mtf}$ & 18 & $\mathrm{sr}$ \\
\hline Chrysolampis mosquitus (Linnaeus, 1758) & beija-flor-vermelho & $g(1), f(2)$ & $\mathrm{ca}$ & $4,6,8$ & aw, s2 \\
\hline Discosura longicaudus (Gmelin, 1788) & bandeirinha & $\begin{array}{l}f(2), c(2)-I N P A \\
2163\end{array}$ & $\mathrm{ca}, \mathrm{mtf}$ & 6,14 & $s 1, s 2, s r$ \\
\hline Chlorostilbon notatus (Reich, 1793) & beija-flor-de-garganta-azul & $\circ(1,2)$ & $\mathrm{ca}$ & 4 & $a w, s 2$ \\
\hline Chlorostilbon mellisugus (Linnaeus, 1758) & esmeralda-de-cauda-azul & $\begin{array}{l}\text { o (2), c (4d) - INPA } \\
2069-2070\end{array}$ & $\mathrm{ca}$ & 6 & s2 \\
\hline Thalurania furcata (Gmelin, 1788) & beija-flor-tesoura-verde & $g(2)$ & $\mathrm{mtf}$ & $12,13,17$ & $\mathrm{sr}$ \\
\hline Hylocharis sapphirina (Gmelin, 1788) & beija-flor-safira & $g(2)$ & $\mathrm{ca}$ & 24 & $\mathrm{sr}$ \\
\hline Hylocharis cyanus (Vieillot, 1818) & beija-flor-roxo & $g(1)$ & $\mathrm{ca}$ & 4,24 & $a w, s r$ \\
\hline Polytmus theresiae (Da Silva Maia, 1843) & beija-flor-verde & $\begin{array}{l}g(1,2), f(2), c(4 d) \\
- \text { INPA 2062-68 }\end{array}$ & $\mathrm{ca}$ & $2-6,8,11,19$ & $a w, s 1, s 2$ \\
\hline Amazilia versicolor (Vieillot, 1818) & beija-flor-de-banda-branca & $f(2), o(1)$ & $\mathrm{ca}$ & 4 & aw, s2 \\
\hline Amazilia fimbriata (Gmelin, 1788) & beija-flor-de-garganta-verde & $f(2), o(1)$ & $\mathrm{ca}$ & $4,7,10,20$ & $a w, s 1, s 2, s r$ \\
\hline Amazilia viridigaster (Bourcier, 1843) & beija-flor-de-barriga-verde & $f(2), o(2)$ & cal, ca & 2,24 & $s 1, s 2, s r$ \\
\hline Heliothryx auritus (Gmelin, 1788) & beija-flor-de-bochecha-azul & $\circ(2)$ & $\mathrm{mtf}$ & 12,18 & $\mathrm{sr}$ \\
\hline $\begin{array}{l}\text { Heliomaster longirostris (Audebert \& } \\
\text { Vieillot, 1801) }\end{array}$ & bico-reto-cinzento & $\circ(1)$ & $\mathrm{ca}$ & 4 & aw \\
\hline Calliphlox amethystina (Boddaert, 1783) & estrelinha-ametista & $\circ(1,2)$ & $\mathrm{ca}$ & 4,6 & $a w, s 2$ \\
\hline \multicolumn{6}{|l|}{ Trogonidae Lesson, 1828} \\
\hline Trogon melanurus Swainson, 1838 & surucuá-de-cauda-preta & $g(1,2)$ & $\mathrm{mtf}$ & $4,15,16$ & aw, sr \\
\hline Trogon viridis Linnaeus, 1766 & $\begin{array}{l}\text { surucuá-grande-de-barriga- } \\
\text { amarela }\end{array}$ & $g(1,2), f(2)$ & $\mathrm{mtf}, \mathrm{ca}, \mathrm{cp}$, ig & $3-6,8,12,15-22,24,25$ & $a w, s 1, s 2, s r$ \\
\hline Trogon violaceus Gmelin, 1788 & surucuá-violáceo & $g(1)$ & $\mathrm{ca}$ & 4 & aw \\
\hline Trogon curucui Linnaeus, 1766 & surucuá-de-barriga-vermelha & $g(2)$ & ca, ig & 11 & s2 \\
\hline Trogon personatus Gould, 1842 & surucuá-mascarado & $g(2)$ & $\mathrm{mm}$ & 2 & s2 \\
\hline \multicolumn{6}{|l|}{ Alcedinidae Rafinesque, 1815} \\
\hline Megaceryle torquata (Linnaeus, 1766) & martim-pescador-grande & $g(1,2)$ & $\mathrm{r}, \mathrm{ca}$ & $1,4,20$ & $a w, s 2, s r$ \\
\hline Chloroceryle amazona (Latham, 1790) & martim-pescador-verde & $\circ(1)$ & $\mathrm{r}$ & $1,4,20$ & $a w, s 2, s r$ \\
\hline Chloroceryle aenea (Pallas, 1764) & martim-pescador-verde & $\circ(2)$ & $\mathrm{r}, \mathrm{ca}$ & 10 & sc \\
\hline Chloroceryle americana (Gmelin, 1788) & martim-pescador-pequeno & $\circ(1)$ & r & 1,4 & $a w, s 2, s r$ \\
\hline Chloroceryle inda (Linnaeus, 1766) & martim-pescador-da-mata & $\circ(1)$ & $r$ & 4 & aw \\
\hline \multicolumn{6}{|l|}{ Momotidae Gray, 1840} \\
\hline Momotus momota (Linnaeus, 1766) & udu-de-coroa-azul & $g(1,2), f(2)$ & $\mathrm{mtf}, \mathrm{cp}$ & $1,4,16-18,21,22$ & $a w, s r$ \\
\hline
\end{tabular}


Appendix 1. Continued.

\begin{tabular}{|c|c|c|c|c|c|}
\hline Taxons & Portuguese common name & Evidences $^{1}$ & Habitats $^{2}$ & Sites $^{3}$ & Sources ${ }^{4}$ \\
\hline \multicolumn{6}{|l|}{ Galbulidae Vigors, 1825} \\
\hline Galbula albirostris Latham, 1790 & ariramba-de-bico-amarelo & $g(2), f(2)$ & $\mathrm{mtf}$ & $13,17,25$ & sr \\
\hline Galbula galbula (Linnaeus, 1766) & ariramba-de-cauda-verde & $g(1), f(2)$ & ig & 4,20 & $a w, s 2, s r$ \\
\hline Galbula leucogastra Vieillot, 1817 & ariramba-bronzeada & $g(1,2), f(2)$ & са & $3-6,10,24$ & $a w, s 1, s 2, s r$ \\
\hline Galbula dea (Linnaeus, 1758) & ariramba-do-paraíso & $g(1,2)$ & $\mathrm{mtf}$ & $4,15-18,20,22,26$ & aw, sr \\
\hline Jacamerops aureus (Statius Muller, 1776) & jacamaraçu & $g(1)$ & $\mathrm{mtf}$ & $4,15,16,18$ & $\mathrm{aw}, \mathrm{sr}$ \\
\hline \multicolumn{6}{|l|}{ Bucconidae Horsfield, 1821} \\
\hline Notharchus macrorhynchos (Gmelin, 1788) & macuru-de-testa-branca & $g(2)$ & $\mathrm{mtf}$ & 25 & sr \\
\hline Bucco tamatia Gmelin, 1788 & rapazinho-carijó & $g(1,2), f(2)$ & са & $1,4-8,22,24$ & $a w, s 2, s r$ \\
\hline Bucco capensis Linnaeus, 1766 & rapazinho-de-colar & $g(1)$ & $\mathrm{ca}, \mathrm{mtf}$ & 4 & aw \\
\hline Monasa atra (Boddaert, 1783) & chora-chuva-de-asa-branca & o (2) & $\mathrm{mtf}$ & 1 & sr \\
\hline Monasa nigrifrons (Spix, 1824) & chora-chuva-preto & $g(1)$ & ig & $1,4,20$ & aw, sr \\
\hline Chelidoptera tenebrosa (Pallas, 1782) & urubuzinho & $g(1), o(2)$ & ig, ca & $1,3,4,6,19,20,24$ & $a w, s 2, s r$ \\
\hline \multicolumn{6}{|l|}{ Capitonidae Bonaparte, 1838} \\
\hline Capito auratus (Dumont, 1816) & capitão-de-fronte-dourada & $g(1,2)$ & $\mathrm{mtf}$ & $4,15,16,18,20,22$ & aw, sr \\
\hline \multicolumn{6}{|l|}{ Ramphastidae Vigors, 1825} \\
\hline Ramphastos tucanus Linnaeus, 1758 & tucano-grande-de-papo-branco & $g(1,2), o(2)$ & $\mathrm{ca}, \mathrm{ig}, \mathrm{mtf}$ & $4,12,15-18,20-22,24,25$ & $a w, s 2, s r$ \\
\hline Ramphastos vitellinus Lichtenstein, 1823 & tucano-de-bico-preto & $g(2), f(3), o(1,2)$ & $\mathrm{ca}, \mathrm{ig}, \mathrm{mtf}$ & $4,15-18,22,25,26$ & $a w, s 2, s r$ \\
\hline Selenidera nattereri (Gould, 1836) & saripoca-de-bico-castanho & $g(1,2)$ & $\mathrm{mtf}$ & 4,22 & aw, sr \\
\hline Pteroglossus viridis (Linnaeus, 1766) & araçari-miudinho & $g(1), f(2)$ & са & 3,27 & $a w, s 1$ \\
\hline Pteroglossus azara (Vieillot, 1819)) & araçari-de-bico-de-marfim & $g(1)$ & са & 4 & aw \\
\hline Pteroglossus aracari (Linnaeus, 1758) & araçari-de-bico-branco & $\vee(3)$ & ca & 24 & sr \\
\hline Pteroglossus pluricinctus Gould, 1835 & araçari-de-cinta-dupla & $\circ(2)$ & $\mathrm{mtf}$ & 17 & sr \\
\hline \multicolumn{6}{|l|}{ Picidae Leach, 1820} \\
\hline $\begin{array}{l}\text { Picumnus pumilus Cabanis \& Heine, 1863/P. } \\
\text { lafresnayi Malherbe, } 1862 \text { ) }\end{array}$ & pica-pau-anão-do-amazonas & o (2) & ca & 6 & s2 \\
\hline Picumnus exilis (Lichtenstein, 1823) & pica-pau-anão-de-pintas-amarelas & $\begin{array}{l}o(1), c(4 d)-I N P A \\
2061\end{array}$ & ca & 4 & aw \\
\hline Melanerpes cruentatus (Boddaert, 1783) & benedito-de-testa-vermelha & $\vee(2)$ & $\mathrm{ca}, \mathrm{ig}, \mathrm{cp}$ & $17,18,20,21,25,26$ & sr \\
\hline Veniliornis affinis (Swainson, 1821) & picapauzinho-avermelhado & $g(1,2)$ & $\mathrm{mtf}$ & 4,15 & $\mathrm{aw}, \mathrm{sr}$ \\
\hline Piculus flavigula (Boddaert, 1783) & pica-pau-bufador & $g(2), v(1)$ & $\mathrm{mtf}$ & $4,17,18$ & $\mathrm{aw}, \mathrm{sr}$ \\
\hline Piculus chrysochloros (Vieillot, 1818) & pica-pau-dourado-escuro & $g(2)$ & $\mathrm{ca}, \mathrm{mtf}$ & 20 & s2, sr \\
\hline Colaptes punctigula (Boddaert, 1783) & pica-pau-de-peito-pontilhado & o $(2,3)$ & ig & 20 & sr \\
\hline Celeus grammicus (Natterer \& Malherbe, 1845) & picapauzinho-chocolate & $\begin{array}{l}g(2), v(1), c(4 d)- \\
\text { INPA } 1329\end{array}$ & mtf, ca & $4,9,16$ & $a w, s 2, s r$ \\
\hline Celeus elegans (Statius Muller, 1776) & pica-pau-chocolate & $g(1,2)$ & $\mathrm{mtf}, \mathrm{ca}$ & $4,5,17-19$ & $a w, s 2, s r$ \\
\hline Celeus flavus (Statius Muller, 1776) & pica-pau-amarelo & $g(1), v(2)$ & $\mathrm{ca}$, ig & $4,7,17$ & $a w, s 2, s r$ \\
\hline Celeus torquatus (Boddaert, 1783) & pica-pau-de-coleira & $g(2), f(2)$ & $\mathrm{mtf}, \mathrm{ca}$ & $4,9,16,24$ & $a w, s 2, s r$ \\
\hline Dryocopus lineatus (Linnaeus, 1766) & pica-pau-de-banda-branca & $g(1), v(2)$ & ig, ca & $4,11,19,20$ & $a w, s 2, s r$ \\
\hline Campephilus rubricollis (Boddaert, 1783) & pica-pau-de-barriga-vermelha & $o(1), v(2)$ & $\mathrm{mtf}, \mathrm{ca}$ & $4,9,17$ & $a w, s 2, s r$ \\
\hline Campephilus melanoleucos (Gmelin, 1788) & pica-pau-de-topete-vermelho & $g(1,2)$ & ig, mtf, ca & $1,4,20$ & aw, s2, sr \\
\hline \multicolumn{6}{|l|}{ Falconidae Leach, 1820} \\
\hline Daptrius ater Vieillot, 1816 & gavião-de-anta & $g(1), o(2)$ & ig & $1,4,20,24$ & $a w, s 2, s r$ \\
\hline Ibycter americanus (Boddaert, 1783) & gralhão & $g(1)$ & ig, mtf & 1,4 & $a w, s r$ \\
\hline Caracara plancus (Miller, 1777) & caracará & o (1) & са & 4 & aw \\
\hline
\end{tabular}


Appendix 1. Continued.

\begin{tabular}{|c|c|c|c|c|c|}
\hline Taxons & Portuguese common name & Evidences $^{1}$ & Habitats $^{2}$ & Sites $^{3}$ & Sources ${ }^{4}$ \\
\hline Milvago chimachima (Vieillot, 1816) & carrapateiro & $o(1,2)$ & ig, ca, cp & $1,4,6,10,11,19$ & $a w, s 2, s r$ \\
\hline Herpetotheres cachinnans (Linnaeus, 1758) & acauã & $v(2)$ & $\mathrm{ca}$ & 1 & $\mathrm{sr}$ \\
\hline Micrastur gilvicollis (Vieillot, 1817) & falcão-mateiro & $g(2)$ & $\mathrm{mtf}$ & 15 & $\mathrm{sr}$ \\
\hline Micrastur mirandollei (Schlegel, 1862) & tanatau & $g(2)$ & ig & 11 & s2 \\
\hline Micrastur semitorquatus (Vieillot, 1817) & falcão-relógio & $g(1,2)$ & $\mathrm{ca}$ & 4,24 & $a w, s r$ \\
\hline Falco rufigularis Daudin, 1800 & cauré & $o(1,2)$ & ig & 1,4 & $a w, s 2, s r$ \\
\hline \multicolumn{6}{|l|}{ Psittacidae Rafinesque, 1815} \\
\hline Ara ararauna (Linnaeus, 1758) & arara-canindé & $g(1), f(2)$ & $\mathrm{mtf}, \mathrm{ig}, \mathrm{ca}$ & $1,3,4,17,18,20,22,24$ & $a w, s 2, s r$ \\
\hline Ara macao (Linnaeus, 1758) & araracanga & $o(1), f(2)$ & $\mathrm{mtf}$, ig & 17,18 & $a w, s r$ \\
\hline Ara chloropterus Gray, 1859 & arara-vermelha-grande & $g(1), o(2)$ & $\mathrm{mtf}$ & 17 & $a w, s 2, s r$ \\
\hline Orthopsittaca manilatus (Boddaert, 1783) & maracanã-do-buriti & $o(1,2)$ & ig, ca & $1,4,6$ & $a w, s 2, s r$ \\
\hline Psittacara leucophthalmus (Statius Muller, 1776) & periquitão-maracanã & $\vee(2)$ & ig & 1,20 & sr \\
\hline Eupsittula pertinax (Linnaeus, 1758) & periquito-de-bochecha-parda & $g(1,2), o(2)$ & ig, ca & $1,4,5,7-11,19$ & $a w, s 2, s r$ \\
\hline Pyrrhura egregia (Sclater, 1881) & tiriba-de-cauda-roxa & $\vee(2)$ & $\mathrm{mm}$ & 2 & s2 \\
\hline Brotogeris chrysoptera (Linnaeus, 1766) & periquito-de-asa-dourada & $g(1,2)$ & $\mathrm{mtf}, \mathrm{ig}, \mathrm{ca}$ & 1,4 & $a w, s r$ \\
\hline Brotogeris sanctithomae (Statius Muller, 1776) & periquito-testinha & o (1) & ig & $1,4,20$ & aw \\
\hline Touit purpuratus (Gmelin, 1788) & apuim-de-costas-azuis & $g(1)$ & $\mathrm{mtf}$ & $1,4,12$ & $a w, s r$ \\
\hline Pionites melanocephalus (Linnaeus, 1758) & marianinha-de-cabeça-preta & $g(1,2), f(2)$ & $\mathrm{mtf}, \mathrm{ig}, \mathrm{ca}$ & $4,12,15,17,18,20,24,25$ & $a w, s 1, s 2, s r$ \\
\hline Pyrilia barrabandi (Kuhl, 1820) & curica-de-bochecha-laranja & $g(1,2)$ & $\mathrm{mtf}, \mathrm{ca}$ & $1,4,15-18,20,22$ & aw, sr \\
\hline Pionus menstruus (Linnaeus, 1766) & maitaca-de-cabeça-azul & $g(2), o(1)$ & $\mathrm{mtf}$, ig & $4,12,17,18,20,22,24$ & $\mathrm{aw}, \mathrm{sr}$ \\
\hline Pionus fuscus (Statius Muller, 1776) & maitaca-roxa & $g(1,2)$ & $\mathrm{mtf}, \mathrm{ca}$ & $\begin{array}{l}4,12,15,17,18,21,24,- \\
25\end{array}$ & $a w, s r$ \\
\hline Amazona festiva (Linnaeus, 1758) & papagaio-da-várzea & $o(1,2)$ & ig & $4,20,22$ & $a w, s 2, s r$ \\
\hline Amazona amazonica (Linnaeus, 1766) & curica & $g(1,2), f(2)$ & ig, ca & $1,4,6,7,9,11,19,20$ & $a w, s 2, s r$ \\
\hline Amazona farinosa (Boddaert, 1783) & papagaio-moleiro & $g(2), o(1)$ & $\mathrm{mtf}, \mathrm{ca}$ & $4,12,15,17,18$ & $\mathrm{aw}, \mathrm{sr}$ \\
\hline \multicolumn{6}{|l|}{ Thamnophilidae Swainson, 1824} \\
\hline Euchrepomis spodioptila (Sclater \& Salvin, 1881) & zidedê-de-asa-cinza & $g(2)$ & $\mathrm{mtf}$ & 15,16 & sr \\
\hline Pygiptila stellaris (Spix, 1825) & choca-cantadora & $g(1,2)$ & ig, $\mathrm{cp}$ & $4,15,21,22$ & $a w, s r$ \\
\hline Ammonastes pelzelni (Sclater, 1890) & formigueiro-de-barriga-cinza & $g(2), f(2)$ & $\mathrm{ca}$ & 24 & sr \\
\hline Myrmophylax atrothorax (Boddaert, 1783) & formigueiro-de-peito-preto & $g(1,2), v(2)$ & $\mathrm{mtf}, \mathrm{cp}$ & $4,16-18,22,26$ & $a w, s 2, s r$ \\
\hline Aprositornis disjuncta (Friedmann, 1945) & formigueiro-de-yapacana & c (4d) - INPA 2081 & ca & 10 & sc \\
\hline Myrmeciza longipes (Swainson, 1825) & formigueiro-de-barriga-branca & $g(2), f(2)$ & $\mathrm{mtf}, \mathrm{mm}$ & $12,17,18$ & s2, sr \\
\hline Epinecrophylla haematonota (Sclater, 1857) & choquinha-de-garganta-carijó & $g(2), v(1)$ & $\mathrm{mtf}$ & $4,12,15,17,18,25$ & aw, sr \\
\hline Myrmotherula brachyura (Hermann, 1783) & choquinha-miúda & $g(1,2)$ & $\mathrm{mtf}$ & $1,4,16,20,21$ & $a w, s r$ \\
\hline Myrmotherula ambigua Zimmer, 1932 & choquinha-de-coroa-listrada & $g(2)$ & $\mathrm{mtf}, \mathrm{mm}$ & $1,15,18$ & sr \\
\hline Myrmotherula cherriei Berlepsch \& Hartert, 1902 & choquinha-de-peito-riscado & $g(1,2), v(2)$ & $\mathrm{ca}$ & $4,6,24$ & $a w, s 2, s r$ \\
\hline Myrmotherula axillaris (Vieillot, 1817) & choquinha-de-flanco-branco & $g(2), v(1)$ & $\mathrm{mtf}$ & $4,15,25$ & $a w, s r$ \\
\hline Myrmotherula longipennis Pelzeln, 1868 & choquinha-de-asa-comprida & $g(2), o(1)$ & $\mathrm{mtf}$ & $4,15,16,21,25$ & $a w, s r$ \\
\hline Myrmotherula menetriesii (d'Orbigny, 1837) & choquinha-de-garganta-cinza & $g(1,2)$ & $\mathrm{mtf}$ & $4,17,18$ & $a w, s r$ \\
\hline Formicivora grisea (Boddaert, 1783) & papa-formiga-pardo & $\begin{array}{l}g(1,2), f(2), c(4 d) \\
- \text { INPA 2094-2102 }\end{array}$ & ca & $4-11,19,24$ & $a w, s 1, s 2, s r$ \\
\hline Thamnomanes ardesiacus (Sclater \& Salvin, 1867) & uirapuru-de-garganta-preta & $g(2)$ & $\mathrm{mtf}$ & $17,18,21$ & $\mathrm{sr}$ \\
\hline Thamnomanes caesius (Temminck, 1820) & ipecuá & $g(1,2)$ & $\mathrm{mtf}$ & $4,15-18,21,22,24,25$ & $a w, s r$ \\
\hline Megastictus margaritatus (Sclater, 1855) & choca-pintada & $g(1,2), f(2)$ & $\mathrm{mtf}$ & $4,16,21,25$ & $a w, s r$ \\
\hline Herpsilochmus dorsimaculatus Pelzeln, 1868 & chorozinho-de-costas-manchadas & $g(1,2)$ & $\mathrm{mtf}, \mathrm{ca}$ & $4,15-18,22,24,26$ & $a w, s r$ \\
\hline
\end{tabular}

ZOOLOGIA 31 (4): 343-360, August, 2014 
Appendix 1. Continued.

\begin{tabular}{|c|c|c|c|c|c|}
\hline Taxons & Portuguese common name & Evidences $^{1}$ & Habitats $^{2}$ & Sites $^{3}$ & Sources $^{4}$ \\
\hline Sakesphorus canadensis (Linnaeus, 1766) & choca-de-crista-preta & $\mathrm{g}(1,2), \mathrm{o}(2)$ & ig, ca & $4,19,20$ & $\mathrm{aw}, \mathrm{s} 2, \mathrm{sr}$ \\
\hline Thamnophilus doliatus (Linnaeus, 1764) & choca-barrada & $\begin{array}{l}\mathrm{g}(1,2), \mathrm{f}(2), \mathrm{c}(2)- \\
\text { INPA } 2433-34\end{array}$ & $\mathrm{ca}, \mathrm{cal}$ & $2,4,6-8,10,11,19$ & aw, s2 \\
\hline Thamnophilus murinus Sclater \& Salvin, 1868 & choca-murina & $g(1,2)$ & mtf, cp & $4,12-18,21,22,25$ & $a w, s 2, s r$ \\
\hline Thamnophilus nigrocinereus Sclater, 1855 & choca-preta-e-cinza & $g(1)$ & ig & 4 & aw \\
\hline Thamnophilus aethiops Sclater, 1858 & choca-lisa & $g(2), v(1)$ & $\mathrm{mtf}$ & $4,12,14-18,21,22$ & aw, sr \\
\hline Thamnophilus amazonicus Sclater, 1858 & choca-canela & $g(1,2)$ & $\mathrm{ca}$ & $3,4,7-9,23,24$ & $a w, s 2, s r$ \\
\hline Cymbilaimus lineatus (Leach, 1814) & papa-formiga-barrado & $g(1,2)$ & $\mathrm{mtf}$ & $1,4,17,18$ & $a w, s r$ \\
\hline Taraba major (Vieillot, 1816) & choró-boi & $g(2)$ & ig, $\mathrm{cp}$ & 22 & $\mathrm{sr}$ \\
\hline Sclateria naevia (Gmelin, 1788) & papa-formiga-do-igarapé & $g(2), \circ(1)$ & ig & $4,16,20$ & $a w, s r$ \\
\hline Hypocnemoides melanopogon (Sclater, 1857) & solta-asa-do-norte & $g(1,2)$ & ig & $1,4,20$ & $a w, s r$ \\
\hline Hylophylax naevius (Gmelin, 1789) & guarda-floresta & $g(1,2)$ & $\mathrm{mtf}$ & $4,15,17$ & $a w, s r$ \\
\hline Percnostola minor Pelzeln, 1868 & formigueiro-de-pelzeln & $g(1), f(2)$ & $\mathrm{mtf}$ & $4,13,17,18$ & $a w, s r$ \\
\hline Myrmoborus leucophrys (Tschudi, 1844) & papa-formiga-de-sobrancelha & $g(2), f(2)$ & $\mathrm{mtf}$ & 21 & $\mathrm{sr}$ \\
\hline Cercomacra cinerascens (Sclater, 1857) & chororó-pocuá & $g(2)$ & $\mathrm{mtf}$ & 18,24 & $\mathrm{sr}$ \\
\hline Cercomacra tyrannina (Sclater, 1855) & chororó-escuro & $g(2), f(2)$ & $\begin{array}{l}\mathrm{ca}, \mathrm{ig}, \mathrm{cp}, \\
\mathrm{mm}\end{array}$ & 2 & s2 \\
\hline Hypocnemis flavescens Sclater, 1864 & cantador-sulfúreo & $g(2), f(2), v(2)$ & $\mathrm{mtf}, \mathrm{cp}$ & $13-18,21,22,25$ & $s 2, s r$ \\
\hline Pithys albifrons (Linnaeus, 1766) & papa-formiga-de-topete & $g(1,2), f(3)$ & $\mathrm{mtf}$ & $4,12,13,16-18,21,22$ & aw, sr \\
\hline Willisornis poecilinotus (Cabanis, 1847) & rendadinho & $g(1,2), f(2)$ & $\mathrm{mtf}, \mathrm{ca}$ & $4,9,21,22,24$ & $a w, s 1, s 2, s r$ \\
\hline Phlegopsis erythroptera (Gould, 1855) & mãe-de-taoca-avermelhada & $g(1,2)$ & $\mathrm{mtf}, \mathrm{ca}$ & 4,22 & $a w, s 2, s r$ \\
\hline Gymnopithys rufigula (Boddaert, 1783) & \multicolumn{2}{|c|}{ mãe-de-taoca-de-garganta-vermelha g $(1,2)$} & $\mathrm{mtf}$ & $4,12,15,16,21,22,25$ & $a w, s r$ \\
\hline \multicolumn{6}{|l|}{ Grallariidae Sclater \& Salvin, 1873} \\
\hline Myrmothera campanisona (Hermann, 1783) & tovaca-patinho & $g(2), v(1)$ & $\mathrm{mtf}$ & $4,15,21,25$ & $a w, s r$ \\
\hline \multicolumn{6}{|l|}{ Formicariidae Gray, 1840} \\
\hline Formicarius colma Boddaert, 1783 & galinha-do-mato & $\circ(1)$ & $\mathrm{mtf}, \mathrm{ca}$ & $4,12,15,16,21,22,24$ & aw, sr \\
\hline \multicolumn{6}{|l|}{ Scleruridae Swainson, 1827} \\
\hline Sclerurus macconnelli Chubb, 1919 & vira-folha-de-peito-vermelho & $g(1)$ & $\mathrm{mtf}$ & 4 & aw \\
\hline Sclerurus rufigularis Pelzeln, 1868 & vira-folha-de-bico-curto & $\vee(2)$ & $\mathrm{mtf}$ & 18 & sr \\
\hline \multicolumn{6}{|l|}{ Dendrocolaptidae Gray, 1840} \\
\hline Dendrocincla fuliginosa (Vieillot, 1818) & arapaçu-pardo & $g(1,2), c(4 d)-2075$ & $\mathrm{mtf}, \mathrm{mm}, \mathrm{ca}$ & $4,18,21,22$ & $a w, s 2, s r$ \\
\hline Dendrocincla merula (Lichtenstein, 1829) & arapaçu-da-taoca & $\begin{array}{l}\mathrm{g}(1), \mathrm{c}(4 \mathrm{~d})-\text { INPA } \\
2074\end{array}$ & $\mathrm{mtf}, \mathrm{ca}$ & $4,18,21$ & $a w, s r$ \\
\hline Deconychura longicauda (Pelzeln, 1868) & arapaçu-rabudo & $\vee(2)$ & $\mathrm{mtf}$ & 25 & $\mathrm{sr}$ \\
\hline Sittasomus griseicapillus (Vieillot, 1818) & arapaçu-verde & $g(2)$ & $\mathrm{mtf}, \mathrm{mm}$ & $17,18,25$ & $s 2, s r$ \\
\hline Glyphorynchus spirurus (Vieillot, 1819) & arapaçu-de-bico-de-cunha & $g(2), \circ(1)$ & $\mathrm{mtf}$ & $4,12,13,15-18,21,22$ & aw, sr \\
\hline Nasica longirostris (Vieillot, 1818) & arapaçu-de-bico-comprido & $g(1,2), v(2)$ & ig, ca & $1,4,9,20,22$ & $a w, s 2, s r$ \\
\hline Dendrocolaptes certhia (Boddaert, 1783) & arapaçu-barrado & $g(2), o(1), v(2)$ & $\mathrm{mtf}, \mathrm{ig}, \mathrm{ca}$ & $4,9,15-17$ & $a w, s 2, s r$ \\
\hline Dendrocolaptes picumnus Lichtenstein, 1820 & arapaçu-meio-barrado & $\vee(2)$ & $\mathrm{mtf}$ & 17,18 & sr \\
\hline Dendroplex picus (Gmelin, 1788) & arapaçu-de-bico-branco & $\begin{array}{l}g(1,2), c(4 d)- \\
\text { INPA } 2076\end{array}$ & ig, ca & $1,4,5-7,10,11,19,20,22$ & $a w, s 2, s r$ \\
\hline Dendroplex kienerii (Des Murs, 1855) & arapaçu-ferrugem & $g(2), v(1)$ & ig, ca & 4 & $a w, s r$ \\
\hline Xiphorhynchus pardalotus (Vieillot, 1818) & arapaçu-assobiador & $g(1,2)$ & $\mathrm{mtf}$ & $4,12,13,15-18,21,24-26$ & $a w, s r$ \\
\hline Xiphorhynchus obsoletus (Lichtenstein, 1820) & arapaçu-riscado & $g(1,2)$ & ig, ca & $4,6,8,20$ & $a w, s 2, s r$ \\
\hline Xiphorhynchus guttatus (Lichtenstein, 1820) & arapaçu-de-garganta-amarela & $g(1,2)$ & $\mathrm{mtf}, \mathrm{cp}$ & $1,4,15,22$ & $a w, s r$ \\
\hline Lepidocolaptes albolineatus (Lafresnaye, 1845) & arapaçu-de-listras-brancas & $g(2), o(1)$ & $\mathrm{mtf}$ & 4 & aw, sr \\
\hline
\end{tabular}


Appendix 1. Continued.

\begin{tabular}{|c|c|c|c|c|c|}
\hline Taxons & Portuguese common name & Evidences $^{1}$ & Habitats $^{2}$ & Sites $^{3}$ & Sources ${ }^{4}$ \\
\hline \multicolumn{6}{|l|}{ Xenopidae Bonaparte, 1854} \\
\hline Xenops minutus (Sparrman, 1788) & bico-virado-miúdo & $g(2)$ & $\mathrm{mtf}$ & 25 & $\mathrm{sr}$ \\
\hline \multicolumn{6}{|l|}{ Furnariidae Gray, 1840} \\
\hline Synallaxis rutilans Temminck, 1823 & joão-teneném-castanho & $g(1,2)$ & $\mathrm{mtf}$ & $1,4,16,21$ & aw, sr \\
\hline Cranioleuca vulpina (Pelzeln, 1856) & arredio-do-rio & $g(2), o(1)$ & ig & $1,4,20$ & $a w, s r$ \\
\hline $\begin{array}{l}\text { Cranioleuca gutturata (d'Orbigny \& } \\
\text { Lafresnaye, 1838) }\end{array}$ & joão-pintado & $\circ(1)$ & ig & 4 & aw \\
\hline Philydor pyrrhodes (Cabanis, 1848) & limpa-folha-vermelho & $g(1,2)$ & $\mathrm{mtf}$ & $4,14,17,18,21,24$ & $a w, s r$ \\
\hline Automolus ochrolaemus (Tschudi, 1844) & barranqueiro-camurça & $g(2)$ & $\mathrm{mtf}, \mathrm{cp}, \mathrm{ca}$, ig & 21,25 & s2, sr \\
\hline Automolus infuscatus (Sclater, 1856) & barranqueiro-pardo & $g(2)$ & $\mathrm{mtf}$ & $13,16,17$ & sr \\
\hline \multicolumn{6}{|l|}{ Pipridae Rafinesque, 1815} \\
\hline Neopelma chrysocephalum (Pelzeln, 1868) & fruxu-do-carrasco & $f(2)$ & $\mathrm{ca}$ & 9 & s1, s2 \\
\hline Tyranneutes stolzmanni (Hellmayr, 1906) & uirapuruzinho & $g(1,2)$ & $\mathrm{mtf}$ & $4,17,18$ & aw, sr \\
\hline Lepidothrix coronata (Spix, 1825) & uirapuru-de-chapéu-azul & $g(1,2)$ & $\mathrm{mtf}, \mathrm{ca}$ & $4,12-18,18,22,24,25$ & aw, sr \\
\hline Lepidothrix suavissima (Salvin \& Godman, 1882) & dançador-do-tepui & $\begin{array}{l}f(2), c(2)-\text { INPA } \\
2435-36\end{array}$ & $\mathrm{~mm}$ & 2 & s2 \\
\hline Xenopipo atronitens Cabanis, 1847 & pretinho & $\begin{array}{l}g(1,2), f(2), c(4 d) \\
\text { - INPA 2050-59 }\end{array}$ & $\mathrm{ca}, \mathrm{mm}$ & $3-10,19,24$ & $a w, s 1, s 2, s r$ \\
\hline Heterocercus flavivertex Pelzeln, 1868 & dançarino-de-crista-amarela & $g(1,2), f(2)$ & ig, ca & 1,4 & $a w, s 1, s 2, s r$ \\
\hline Dixiphia pipra (Linnaeus, 1758) & cabeça-branca & $\begin{array}{l}g(1,2), f(2), c(4 d) \\
- \text { INPA } 2060\end{array}$ & $\mathrm{ca}, \mathrm{mtf}$ & $4,12,16,18,21-26$ & $a w, s 1, s 2, s r$ \\
\hline Pipra filicauda Spix, 1825 & rabo-de-arame & $\vee(1)$ & ig & 4 & aw \\
\hline Ceratopipra cornuta (Spix, 1825) & dançador-de-crista & $\begin{array}{l}f(2), c(2)-\text { INPA } \\
2437\end{array}$ & $\mathrm{~mm}$ & 2 & s2 \\
\hline Ceratopipra erythrocephala (Linnaeus, 1758) & cabeça-de-ouro & $g(2), v(1)$ & $\mathrm{mtf}, \mathrm{ca}, \mathrm{cp}$, ig & $4,16-18,20,21,24-26$ & aw, s2, sr \\
\hline \multicolumn{6}{|l|}{ Onychorhynchidae Tello et al., 2009} \\
\hline Onychorhynchus coronatus (Statius Muller, 1776) & maria-leque & $\mathrm{o}(2,3), \mathrm{v}(2)$ & $\mathrm{mtf}$ & 14,18 & $\mathrm{sr}$ \\
\hline Terenotriccus erythrurus (Cabanis, 1847) & papa-moscas-uirapuru & $g(2)$ & $\mathrm{mtf}$ & $1,15,16,18$ & $\mathrm{sr}$ \\
\hline Myiobius barbatus (Gmelin, 1789) & assanhadinho & $g(1,2)$ & $\mathrm{mtf}$ & 4,18 & $a w, s r$ \\
\hline \multicolumn{6}{|l|}{ Tityridae Gray, 1840} \\
\hline Schiffornis major Des Murs, 1856 & flautim-ruivo & $g(1,2)$ & ig & 1,4 & $a w, s r$ \\
\hline Schiffornis turdina (Wied, 1831) & flautim-marrom & $g(2), v(1)$ & $\mathrm{mtf}, \mathrm{ca}$ & $4,9,12,16,18,24$ & aw, s2, sr \\
\hline Laniocera hypopyrra (Vieillot, 1817) & chorona-cinza & $g(2), o(1)$ & $\mathrm{mtf}$ & $4,15,25$ & $a w, s r$ \\
\hline Pachyramphus rufus (Boddaert, 1783) & caneleiro-cinzento & $\circ(2)$ & ig & 20 & sr \\
\hline Pachyramphus marginatus (Lichtenstein, 1823) & caneleiro-bordado & $g(1,2)$ & $\mathrm{mtf}$ & $4,17,18,21$ & aw, s2, sr \\
\hline Pachyramphus surinamus (Linnaeus, 1766) & caneleiro-da-guiana & $g(1,2)$ & $\mathrm{mtf}$ & 4,15 & $a w, s r$ \\
\hline Pachyramphus minor (Lesson, 1830) & caneleiro-pequeno & $\circ(1)$ & $\mathrm{mtf}$ & $4,17,18$ & aw, sr \\
\hline \multicolumn{6}{|l|}{ Cotingidae Bonaparte, 1849} \\
\hline Cotinga cayana (Linnaeus, 1766) & anambé-azul & $f(2), o(1)$ & ig & $1,4,20$ & aw, sr \\
\hline Lipaugus vociferans (Wied, 1820) & cricrió & $g(1,2)$ & $\mathrm{mtf}$, ig, ca & $4,15,16,17,18,20,21,22,25$ & aw, s2, sr \\
\hline Xipholena punicea (Pallas, 1764) & anambé-pompadora & $g(1), o(2), v(2)$ & $\mathrm{ca}, \mathrm{mtf}$ & $1,3,4,16$ & aw, s2, sr \\
\hline Gymnoderus foetidus (Linnaeus, 1758) & anambé-pombo & $\circ(1,2)$ & ig & 4,20 & aw, s2, sr \\
\hline Cephalopterus ornatus Geoffroy Saint-Hilaire, 1809 & anambé-preto & $f(2), o(1)$ & ig & 4,20 & aw, sr \\
\hline Rupicola rupicola (Linnaeus, 1766) & galo-da-serra & $f(4 a)$ & $\mathrm{mm}$ & 14 & sc \\
\hline \multicolumn{6}{|l|}{ Pipritidae Ohlson et al., 2013} \\
\hline Piprites chloris (Temminck, 1822) & papinho-amarelo & $g(2)$ & $\mathrm{mtf}, \mathrm{ca}$ & $9,15-18,21$ & s2, sr \\
\hline
\end{tabular}


Appendix 1. Continued.

\begin{tabular}{|c|c|c|c|c|c|}
\hline Taxons & Portuguese common name & Evidences $^{1}$ & Habitats $^{2}$ & Sites $^{3}$ & Sources $^{4}$ \\
\hline \multicolumn{6}{|l|}{ Platyrinchidae Bonaparte, 1854} \\
\hline Platyrinchus coronatus Sclater, 1858 & patinho-de-coroa-dourada & $f(2)$ & $\mathrm{mtf}$ & 12 & sr \\
\hline Platyrinchus platyrhynchos (Gmelin, 1788) & patinho-de-coroa-branca & $g(1,2)$ & $\mathrm{mtf}$ & 4,16 & $a w, s r$ \\
\hline Neopipo cinnamomea (Lawrence, 1869) & enferrujadinho & $g(1)$ & ca & 4 & aw \\
\hline \multicolumn{6}{|l|}{ Rhynchocyclidae Berlepsch, 1907} \\
\hline Mionectes oleagineus (Lichtenstein, 1823) & abre-asa & $\circ(1)$ & $\mathrm{mtf}, \mathrm{cp}, \mathrm{ca}$ & $4,12,14-18,21,24$ & aw, sr \\
\hline Mionectes macconnelli (Chubb, 1919) & abre-asa-da-mata & $g(2)$ & $\mathrm{mtf}, \mathrm{ca}$ & 25 & $\mathrm{sr}$ \\
\hline Corythopis torquatus Tschudi, 1844 & estalador-do-norte & $g(2), v(1)$ & $\mathrm{mtf}$ & 4 & aw, sr \\
\hline Tolmomyias sulphurescens (Spix, 1825) & bico-chato-de-orelha-preta & $\vee(2)$ & ig & 1,20 & $\mathrm{sr}$ \\
\hline Tolmomyias assimilis (Pelzeln, 1868) & bico-chato-da-copa & $g(1,2)$ & $\mathrm{mtf}$ & $4,12,15-18,22$ & $a w, s r$ \\
\hline Tolmomyias poliocephalus (Taczanowski, 1884) & bico-chato-de-cabeça-cinza & $g(2), v(1)$ & $\mathrm{mtf}, \mathrm{cp}, \mathrm{ca}$, ig & $4,15,16,18,20-22$ & $\mathrm{aw}, \mathrm{sr}$ \\
\hline Tolmomyias flaviventris (Wied, 1831) & bico-chato-amarelo & $\begin{array}{l}g(1,2), c(4 d)- \\
\text { INPA } 2087\end{array}$ & $\mathrm{ca}$ & 4,6 & $a w, s 2$ \\
\hline Todirostrum maculatum (Desmarest, 1806) & ferreirinho-estriado & $g(1,2)$ & ig, ca & $1,4,20$ & aw, sr \\
\hline Todirostrum pictum Salvin, 1897 & ferreirinho-pintado & $g(2)$ & $\mathrm{mtf}, \mathrm{cp}$ & $1,15,16,18,22,25,26$ & sr \\
\hline $\begin{array}{l}\text { Myiornis ecaudatus (d'Orbigny \& Lafresnaye, } \\
\text { 1837) }\end{array}$ & caçula & $\vee(1)$ & $\mathrm{mtf}$ & 4 & aw \\
\hline Hemitriccus minor (Snethlage, 1907) & maria-sebinha & $g(1)$ & ig & 4,20 & $a w, s r$ \\
\hline Hemitriccus zosterops (Pelzeln, 1868) & maria-de-olho-branco & $g(2)$ & $\mathrm{mtf}$ & 16 & $\mathrm{sr}$ \\
\hline $\begin{array}{l}\text { Hemitriccus margaritaceiventer (d'Orbigny \& } \\
\text { Lafresnaye, 1837) }\end{array}$ & sebinho-de-olho-de-ouro & $g(2), f(2)$ & $\mathrm{ca}, \mathrm{cal}$ & $2,5-8,10,11,19$ & s2 \\
\hline Hemitriccus inornatus (Pelzeln, 1868) & maria-da-campina & $g(2)$ & ca & 24 & sr \\
\hline Lophotriccus galeatus (Boddaert, 1783) & caga-sebinho-de-penacho & $g(2), f(2), v(1)$ & $\mathrm{mtf}, \mathrm{cp}$ & $4,15,16,22,25$ & aw, sr \\
\hline \multicolumn{6}{|l|}{ Tyrannidae Vigors, 1825} \\
\hline Tyrannulus elatus (Latham, 1790) & maria-te-viu & $g(1,2)$ & $\mathrm{mtf}, \mathrm{cp}, \mathrm{ig}, \mathrm{ca}$ & $3,4,9,15,16,20,21,24,25$ & aw, s2, sr \\
\hline Myiopagis gaimardii (d'Orbigny, 1839) & maria-pechim & $g(1,2)$ & $\mathrm{mtf}, \mathrm{cp}, \mathrm{ig}, \mathrm{ca}$ & $4,15-18,20-22$ & $a w, s 2, s r$ \\
\hline Myiopagis caniceps (Swainson, 1835) & guaracava-cinzenta & $g(2), v(1)$ & $\mathrm{mtf}$ & $4,15,17,18,21,22$ & aw, sr \\
\hline Myiopagis flavivertex (Sclater, 1887) & $\begin{array}{l}\text { guaracava-de-penacho-am- } \\
\text { arelo }\end{array}$ & $g(2), v(1)$ & ig, $\mathrm{cp}$ & 4 & $a w, s r$ \\
\hline Elaenia flavogaster (Thunberg, 1822) & guaracava-de-barriga-amarela & $g(1)$ & ca & 4 & aw \\
\hline Elaenia parvirostris Pelzeln, 1868 & guaracava-de-bico-curto & $\begin{array}{l}\text { c (4d), INPA 2033- } \\
34\end{array}$ & ca & 10 & sc \\
\hline Elaenia cristata Pelzeln, 1868 & $\begin{array}{l}\text { guaracava-de-topete-unifor- } \\
\text { me }\end{array}$ & $\begin{array}{l}g(2), f(2), c(4 d)- \\
\text { INPA 2035-39 }\end{array}$ & $\mathrm{ca}, \mathrm{cal}$ & 2,8 & s2 \\
\hline Elaenia ruficeps Pelzeln, 1868 & $\begin{array}{l}\text { guaracava-de-topete-ver- } \\
\text { melho }\end{array}$ & $\begin{array}{l}g(2), f(2), \text { o }(1,2), c \\
\text { (4d) - INPA 2040-49 }\end{array}$ & $\mathrm{ca}, \mathrm{cal}$ & $3,4-11,19,24$ & $a w, s 1, s 2, s r$ \\
\hline Ornithion inerme Hartlaub, 1853 & poiaeiro-de-sobrancelha & $g(1,2)$ & $\mathrm{mtf}$ & $4,17,24$ & aw, sr \\
\hline Camptostoma obsoletum (Temminck, 1824) & risadinha & $g(1,2), v(2)$ & ig, ca & $3,4,8,9,20$ & $a w, s 2, s r$ \\
\hline Capsiempis flaveola (Lichtenstein, 1823) & marianinha-amarela & $g(1,2)$ & ig & 4 & aw, sr \\
\hline Zimmerius gracilipes (Sclater \& Salvin, 1868) & poiaeiro-de-pata-fina & $g(1,2)$ & $\mathrm{mtf}, \mathrm{ig}, \mathrm{ca}$ & $4,15,20$ & $a w, s 2, s r$ \\
\hline Inezia subflava (Sclater \& Salvin, 1873) & amarelinho & $\circ(1,2), v(2)$ & ig, ca & 3,4 & aw, s2 \\
\hline Hirundinea ferruginea (Gmelin, 1788) & gibão-de-couro & $f(4 c), o(3)$ & cal & 2 & s2, sr, ter \\
\hline Lathrotriccus euleri (Cabanis, 1868) & enferrujado & $\circ(1)$ & ig & 4 & aw \\
\hline Cnemotriccus fuscatus (Wied, 1831) & guaracavuçu & $\begin{array}{l}g(2), f(2), c(4 d)- \\
\text { INPA } 2088\end{array}$ & $\mathrm{ca}$ & 6,24 & $s 2, s r$ \\
\hline Ochthornis littoralis (Pelzeln, 1868) & maria-da-praia & $g(2), o(1,2,3)$ & ig & 4,20 & aw, sr \\
\hline Legatus leucophaius (Vieillot, 1818) & bem-te-vi-pirata & $g(1), v(2)$ & ig & 4,20 & $\begin{array}{l}\text { aw, s2, sr } \\
\text { Continues }\end{array}$ \\
\hline
\end{tabular}


Appendix 1. Continued.

\begin{tabular}{|c|c|c|c|c|c|}
\hline Taxons & Portuguese common name & Evidences $^{1}$ & Habitats $^{2}$ & Sites $^{3}$ & Sources $^{4}$ \\
\hline Myiozetetes cayanensis (Linnaeus, 1766) & $\begin{array}{l}\text { bentevizinho-de-asa-ferrug- } \\
\text { ínea }\end{array}$ & $o(1), v(2)$ & $\mathrm{ca}$, ig & $1,3,4,6,19,20,26$ & $a w, s 2, s r$ \\
\hline Pitangus sulphuratus (Linnaeus, 1766) & bem-te-vi & $g(2), o(1,2)$ & $\mathrm{ca}$, ig & $1,3,4,6,8,19-21$ & $a w, s 2, s r$ \\
\hline Philohydor lictor (Lichtenstein, 1823) & bentevizinho-do-brejo & $g(2), o(1,2)$ & ig & 4,20 & $a w, s 2, s r$ \\
\hline Conopias trivirgatus (Wied, 1831) & bem-te-vi-pequeno & $g(1), v(2)$ & ig & 4,20 & $a w, s r$ \\
\hline Conopias parvus (Pelzeln, 1868) & bem-te-vi-da-copa & $g(1,2)$ & $\mathrm{mtf}, \mathrm{ca}$ & $4,12,15-18,21,22,25$ & $a w, s 2, s r$ \\
\hline Myiodynastes maculatus (Statius Muller, 1776) & bem-te-vi-rajado & $\mathrm{o}(2)$ & $\mathrm{mtf}$ & 17,20 & sr \\
\hline Tyrannopsis sulphurea (Spix, 1825) & suiriri-de-garganta-rajada & $\vee(1)$ & ig & 4,22 & $a w, s r$ \\
\hline Empidonomus varius (Vieillot, 1818) & peitica & $o(2)$ & ig & 1 & $\mathrm{sr}$ \\
\hline Tyrannus melancholicus Vieillot, 1819 & suiriri & $f(2), o(1,2,3)$ & ig, ca & $1,4-9,11,19,20,24$ & $a w, s 2, s r$ \\
\hline Tyrannus savana Vieillot, 1808 & tesourinha & $\mathrm{o}(2)$ & ig, ca & 1 & s2 \\
\hline Rhytipterna simplex (Lichtenstein, 1823) & vissiá & $\begin{array}{l}g(1,2), c(4 d)- \\
\text { INPA } 2083\end{array}$ & $\mathrm{mtf}, \mathrm{ca}$ & $4,15-17,22$ & aw, s2, sr \\
\hline Rhytipterna immunda (Sclater \& Salvin, 1873) & vissiá-cantor & $\begin{array}{l}g(1,2), c(4 d)- \\
\text { INPA } 2084\end{array}$ & $\mathrm{ca}$ & 4,11 & aw, s2 \\
\hline $\begin{array}{l}\text { Myiarchus tuberculifer (d'Orbigny \& } \\
\text { Lafresnaye, 1837) }\end{array}$ & maria-cavaleira-pequena & $\begin{array}{l}g(1,2), f(2), c(4 d) \\
- \text { INPA 2085-86 }\end{array}$ & $\mathrm{ca}, \mathrm{cal}, \mathrm{ig}, \mathrm{mtf}$ & $2,4-11,19,20$ & $a w, s 1, s 2, s r$ \\
\hline Myiarchus ferox (Gmelin, 1789) & maria-cavaleira & $g(2), v(1,2)$ & $\mathrm{ca}$, ig & $4,8,20$ & aw, s2, sr \\
\hline Ramphotrigon ruficauda (Spix, 1825) & $\begin{array}{l}\text { bico-chato-de-rabo-verme- } \\
\text { lho }\end{array}$ & $g(1,2)$ & $\mathrm{mtf}, \mathrm{ca}$ & $4,16,21,25$ & $a w, s 2, s r$ \\
\hline Attila cinnamomeus (Gmelin, 1789) & tinguaçu-ferrugem & $g(1), v(2)$ & ig, ca & $4,19,20$ & $a w, s 2, s r$ \\
\hline Attila citriniventris Sclater, 1859 & $\begin{array}{l}\text { tinguaçu-de-barriga-amare- } \\
\text { la }\end{array}$ & $g(2)$ & $\mathrm{ca}$ & 1 & s2, sr \\
\hline Attila spadiceus (Gmelin, 1789) & capitão-de-saíra-amarelo & $g(1,2)$ & $\mathrm{mtf}, \mathrm{ca}$ & $4,9,12,13,16,17,18$ & aw, s2, sr \\
\hline \multicolumn{6}{|l|}{ Vireonidae Swainson, 1837} \\
\hline Cyclarhis gujanensis (Gmelin, 1789) & pitiguari & $g(1,2)$ & $\mathrm{ca}, \mathrm{ig}, \mathrm{cp}$ & $1,4,11,15,19,20,22$ & $a w, s 2, s r$ \\
\hline Vireolanius leucotis (Swainson, 1838) & assobiador-do-castanhal & $v(2,3)$ & $\mathrm{mtf}$ & $12,15,18$ & sr \\
\hline Vireo olivaceus (Linnaeus, 1766) & juruviara & $g(2)$ & $\mathrm{mtf}$ & 15 & sr \\
\hline Hylophilus thoracicus Temminck, 1822 & vite-vite & $\vee(1)$ & $\mathrm{ca}$ & 4 & aw \\
\hline Hylophilus semicinereus Sclater \& Salvin, 1867 & verdinho-da-várzea & $g(1,2)$ & ig & $1,4,20-22,25$ & $a w, s 2, s r$ \\
\hline Hylophilus sclateri Salvin \& Godman, 1883 & vite-vite-do-tepui & $\begin{array}{l}g(2), f(2), c(2)- \\
\text { INPA } 2438\end{array}$ & $\mathrm{~mm}, \mathrm{cal}$ & 2 & s2 \\
\hline Hylophilus brunneiceps Sclater, 1866 & vite-vite-de-cabeça-marrom & $\begin{array}{l}g(1,2), f(2), c(2)- \\
\text { INPA } 2439\end{array}$ & $\mathrm{ca}, \mathrm{cal}$ & $2,4,24$ & $a w, s 2, s r$ \\
\hline $\begin{array}{l}\text { Hylophilus muscicapinus Sclater \& Salvin, } \\
1873\end{array}$ & vite-vite-camurça & $g(1,2)$ & $\mathrm{mtf}$ & $4,15-18,21$ & $a w, s r$ \\
\hline Hylophilus ochraceiceps Sclater, 1860 & vite-vite-uirapuru & $g(1,2)$ & $\mathrm{mtf}$ & $4,12,13,17,18,20,21,24$ & aw, sr \\
\hline \multicolumn{6}{|l|}{ Corvidae Leach, 1820} \\
\hline Cyanocorax heilprini Gentry, 1885 & gralha-de-nuca-azul & $\begin{array}{l}g(2), f(2), \text { o }(2,3) \\
c(4 d) \text { - INPA } 2000\end{array}$ & $\mathrm{ca}$ & 9,24 & s2, sr \\
\hline \multicolumn{6}{|l|}{ Hirundinidae Rafinesque, 1815} \\
\hline Atticora fasciata (Gmelin, 1789) & peitoril & $o(1,2)$ & ig & $1,4,20$ & $a w, s 2, s r$ \\
\hline Stelgidopteryx ruficollis (Vieillot, 1817) & andorinha-serradora & $f(2), o(1,2)$ & ig, ca & 4,5 & $a w, s 2$ \\
\hline Progne tapera (Vieillot, 1817) & andorinha-do-campo & $g(1,2), o(2)$ & ig & 4,20 & $a w, s 2, s r$ \\
\hline Progne subis (Linnaeus, 1758) & andorinha-azul & $\mathrm{o}(2)$ & ig & 20 & sr \\
\hline Progne chalybea (Gmelin, 1789) & $\begin{array}{l}\text { andorinha-doméstica-gran- } \\
\text { de }\end{array}$ & $o(1,2)$ & ig, ca & $1,19,20$ & s2, sr \\
\hline Tachycineta albiventer (Boddaert, 1783) & andorinha-do-rio & $g(1)$ & ig & $1,4,20$ & $a w, s r$ \\
\hline Riparia riparia (Linnaeus, 1758) & andorinha-do-barranco & $\mathrm{o}(1)$ & ig & 4 & aw \\
\hline
\end{tabular}


Appendix 1. Continued.

\begin{tabular}{|c|c|c|c|c|c|}
\hline Taxons & Portuguese common name & Evidences $^{1}$ & Habitats $^{2}$ & Sites $^{3}$ & Sources $^{4}$ \\
\hline \multicolumn{6}{|l|}{ Troglodytidae Swainson, 1831} \\
\hline Microcerculus ustulatus Salvin \& Godman, 1883 & flautista-do-tepui & $v(2)$ & $\mathrm{mm}$ & 2 & s2 \\
\hline Microcerculus bambla (Boddaert, 1783) & uirapuru-de-asa-branca & $g(1,2), f(3)$ & $\mathrm{mtf}$ & $4,12,17,18$ & aw, sr \\
\hline Troglodytes musculus Naumann, 1823 & corruíra & $g(1)$ & $\mathrm{ca}, \mathrm{cp}$ & 4,20 & aw \\
\hline Pheugopedius coraya (Gmelin, 1789) & garrinchão-coraia & $g(1,2)$ & $\mathrm{mtf}, \mathrm{cp}, \mathrm{ca}$ & $\begin{array}{l}4,12-14,16-18,21,22,24- \\
, 25\end{array}$ & aw, s2, sr \\
\hline Cantorchilus leucotis (Lafresnaye, 1845) & $\begin{array}{l}\text { garrinchão-de-barriga-ver- } \\
\text { melha }\end{array}$ & $g(1,2)$ & ig & 4,20 & $\mathrm{aw}, \mathrm{s} 2, \mathrm{sr}$ \\
\hline \multicolumn{6}{|l|}{ Polioptilidae Baird, 1858} \\
\hline Microbates collaris (Pelzeln, 1868) & bico-assovelado-de-coleira & $g(2), f(2), v(1)$ & $\mathrm{mtf}$ & $4,12,16-18$ & $a w, s r$ \\
\hline Ramphocaenus melanurus Vieillot, 1819 & bico-assovelado & $g(2), f(2)$ & $\mathrm{mtf}$, ig & $17,21,25$ & $\mathrm{sr}$ \\
\hline Polioptila plumbea (Gmelin, 1788) & balança-rabo-de-chapéu-preto & $g(1,2)$ & $\mathrm{ca}$, ig & $4,10,19,20$ & $a w, s 2, s r$ \\
\hline \multicolumn{6}{|l|}{ Turdidae Rafinesque, 1815} \\
\hline Turdus leucomelas Vieillot, 1818 & sabiá-barranco & $g(1), f(2)$ & $\mathrm{ca}$ & $4,8,22$ & aw, s1, s2, sr \\
\hline Turdus ignobilis of murinus & caraxué-de-bico-preto & $f(2)$ & cal & 2 & s2 \\
\hline Turdus ignobilis cf arthuri & caraxué-de-bico-preto & $\begin{array}{l}g(1,2), f(2), c(4 d) \\
- \text { INPA 2072-73 }\end{array}$ & $\mathrm{ca}$ & $2,4,5-8,10,11,19,23$ & aw, s1, s2 \\
\hline Turdus albicollis Vieillot, 1818 & sabiá-coleira & $g(2)$ & $\mathrm{mtf}$ & $12,14-18,21,25$ & sr \\
\hline \multicolumn{6}{|l|}{ Mimidae Bonaparte, 1853} \\
\hline Mimus gilvus (Vieillot, 1807) & sabiá-da-praia & $\begin{array}{l}g(2), f(2), c(2)- \\
\text { INPA } 2440\end{array}$ & $\mathrm{ca}$ & 6,8 & s2 \\
\hline \multicolumn{6}{|l|}{ Passerellidae Cabanis \& Heine, 1850} \\
\hline Zonotrichia capensis (Statius Muller, 1776) & tico-tico & $g(2), f(2)$ & $\mathrm{ca}, \mathrm{cal}$ & $2,6,10$ & s2 \\
\hline Arremon taciturnus (Hermann, 1783) & tico-tico-de-bico-preto & $g(2), f(3)$ & $\mathrm{mtf}, \mathrm{cp}$ & $13,14,17,18,21$ & sr \\
\hline \multicolumn{6}{|l|}{ Icteridae Vigors, 1825} \\
\hline Psarocolius viridis (Statius Muller, 1776) & japu-verde & $\circ(1,2)$ & $\mathrm{mtf}, \mathrm{ca}$ & $4,5,8$ & aw, s2 \\
\hline Psarocolius decumanus (Pallas, 1769) & japu & $\circ(2)$ & ig & 1 & s2 \\
\hline Cacicus cela (Linnaeus, 1758) & xexéu & $\circ(1,2)$ & ig, ca & $1,4,20,22$ & $a w, s 2, s r$ \\
\hline Icterus chrysocephalus (Linnaeus, 1766) & rouxinol-do-rio-negro & $g(2), \circ(2)$ & ca & $1,5,7,20,24$ & $\mathrm{~s} 2, \mathrm{sr}$ \\
\hline Molothrus oryzivorus (Gmelin, 1788) & iraúna-grande & $\mathrm{o}(1)$ & ig & 4 & aw \\
\hline \multicolumn{6}{|l|}{ Mitrospingidae Barker et al., 2013} \\
\hline Lamprospiza melanoleuca (Vieillot, 1817) & pipira-de-bico-vermelho & $g(1,2)$ & $\mathrm{mtf}$ & 4,16 & $a w, s r$ \\
\hline \multicolumn{6}{|l|}{ Thraupidae Cabanis, 1847} \\
\hline Coereba flaveola (Linnaeus, 1758) & cambacica & $\begin{array}{l}g(1,2), f(2), c(4 d) \\
\text { - INPA } 2071\end{array}$ & $\mathrm{ca}$ & $1,3-11,19,20$ & $a w, s 1, s 2, s r$ \\
\hline Saltator grossus (Linnaeus, 1766) & bico-encarnado & $g(2)$ & $\mathrm{mtf}$ & 15,21 & $\mathrm{sr}$ \\
\hline Saltator maximus (Statius Muller, 1776) & tempera-viola & $\mathrm{o}(2,3), \mathrm{v}(2)$ & ср & $17,18,20-22$ & sr \\
\hline Schistochlamys melanopis (Latham, 1790) & sanhaçu-de-coleira & $\begin{array}{l}f(2), \text { o }(1,2), c(4 d) \\
\text { - INPA 2090-93 }\end{array}$ & $\mathrm{ca}, \mathrm{cal}$ & $2,3,4,6-8,11,19$ & $a w, s 1, s 2, s r$ \\
\hline Paroaria gularis (Linnaeus, 1766) & cardeal-da-amazônia & $g(1,2)$ & ig & 4,20 & $a w, s r$ \\
\hline Nemosia pileata (Boddaert, 1783) & saíra-de-chapéu-preto & $\circ(2)$ & ig & 20 & sr \\
\hline Lanio penicillatus (Spix, 1825) & pipira-da-taoca & $\circ(2,3)$ & ig & 20 & $\mathrm{sr}$ \\
\hline Lanio cristatus (Linnaeus, 1766) & tiê-galo & $\circ(2,3)$ & $\mathrm{mtf}$ & 16,18 & sr \\
\hline Lanio surinamus (Linnaeus, 1766) & $\begin{array}{l}\text { tem-tem-de-topete-ferrugí- } \\
\text { neo }\end{array}$ & o (1) & $\mathrm{mtf}$ & $4,14,17,18$ & aw, sr \\
\hline Lanio luctuosus (d'Orbigny \& Lafresnaye, 1837) & tem-tem-de-dragona-branca & $\circ(2)$ & $\mathrm{mtf}$ & 12,21 & sr \\
\hline \multirow[t]{2}{*}{ Tachyphonus phoenicius Swainson, 1838} & tem-tem-de-dragona-vermelha & $\begin{array}{l}f(2), o(1,2), c(4 d) \\
- \text { INPA 2023-32 }\end{array}$ & $\mathrm{ca}, \mathrm{cal}$ & $2-4,7-10$ & $a w, s 1, s 2$ \\
\hline & & & & & हоाтाтा \\
\hline
\end{tabular}


Appendix 1. Continued.

\begin{tabular}{|c|c|c|c|c|c|}
\hline Taxons & Portuguese common name & Evidences $^{1}$ & Habitats $^{2}$ & Sites $^{3}$ & Sources $^{4}$ \\
\hline Lanio fulvus (Boddaert, 1783) & pipira-parda & $g(2)$ & $\mathrm{mtf}$ & 12 & $\mathrm{sr}$ \\
\hline Ramphocelus carbo (Pallas, 1764) & pipira-vermelha & $g(1), o(2)$ & ig, $\mathrm{cp}, \mathrm{ca}$ & $4,20,22,24,26$ & $a w, s 2, s r$ \\
\hline Tangara episcopus (Linnaeus, 1766) & sanhaçu-da-amazônia & $o(1,2)$ & ig, ca, cp & 4,20 & aw, s2, sr \\
\hline Tangara palmarum (Wied, 1823) & sanhaçu-do-coqueiro & $\circ(2)$ & ig, $\mathrm{cp}$ & 20 & s2, sr \\
\hline Tangara mexicana (Linnaeus, 1766) & saíra-de-bando & o (1) & $\mathrm{mtf}$ & 4,20 & $a w, s r$ \\
\hline Tangara chilensis (Vigors, 1832) & sete-cores-da-amazônia & $g(2)$ & $\mathrm{mtf}$ & 17,18 & $\mathrm{sr}$ \\
\hline Tangara varia (Statius Muller, 1776) & saíra-carijó & $g(1)$ & $\mathrm{mtf}$ & 4 & aw \\
\hline Tangara gyrola (Linnaeus, 1758) & saíra-de-cabeça-castanha & $\mathrm{o}(2)$ & $\mathrm{mtf}$ & 18 & sr \\
\hline Tangara cayana (Linnaeus, 1766) & saíra-amarela & $\begin{array}{l}\text { o (2), c ( } 4 d) \text { - INPA } \\
2089\end{array}$ & $\mathrm{ca}$ & 19 & s1 \\
\hline Tangara nigrocincta (Bonaparte, 1838) & saíra-mascarada & $\circ(2)$ & $\mathrm{mtf}$ & 17 & sr \\
\hline Tangara velia (Linnaeus, 1758) & saíra-diamante & $g(2)$ & $\mathrm{mtf}$ & 18 & $\mathrm{sr}$ \\
\hline Dacnis flaviventer d'Orbigny \& Lafresnaye, 1837 & saí-amarela & o (1) & ig & 4,20 & aw, sr \\
\hline Dacnis cayana (Linnaeus, 1766) & saí-azul & $g(1), f(2)$ & $\mathrm{mtf}, \mathrm{ca}$ & 4,26 & $a w, s 2, s r$ \\
\hline Cyanerpes nitidus (Hartlaub, 1847) & saí-de-bico-curto & $f(2)$ & $\mathrm{ca}$ & 9 & s1 \\
\hline Cyanerpes caeruleus (Linnaeus, 1758) & saí-de-perna-amarela & o (1) & $\mathrm{mtf}$, ig & 4 & aw \\
\hline Cyanerpes cyaneus (Linnaeus, 1766) & saíra-beija-flor & $\circ(2)$ & $\mathrm{mtf}$, ig & 9 & s2 \\
\hline Chlorophanes spiza (Linnaeus, 1758) & saí-verde & $\circ(2)$ & $\mathrm{mtf}$, ig & 18,21 & sr \\
\hline Hemithraupis flavicollis (Vieillot, 1818) & saíra-galega & $\circ(2)$ & $\mathrm{mtf}$ & $16-18,21$ & $\mathrm{sr}$ \\
\hline Emberizoides herbicola (Vieillot, 1817) & canário-do-campo & $\begin{array}{l}g(2), f(2), c(4 d)- \\
\text { INPA 2078-79 }\end{array}$ & $\mathrm{ca}, \mathrm{cal}$ & $2,5,6,11,19$ & s2, sr \\
\hline Sporophila plumbea (Wied, 1830) & patativa & $\begin{array}{l}g(2), f(2), c(2)- \\
\text { INPA } 2441-43\end{array}$ & $\mathrm{ca}$ & 23 & s2 \\
\hline Sporophila angolensis (Linnaeus, 1766) & curió & $g(1)$ & $\mathrm{ca}$, ig & 4,20 & $a w, s r$ \\
\hline Dolospingus fringilloides (Pelzeln, 1870) & papa-capim-de-coleira & $\begin{array}{l}g(2), f(2) c(4 d)- \\
\text { INPA } 2082\end{array}$ & $\mathrm{ca}$ & 9 & sc \\
\hline \multicolumn{6}{|l|}{ Cardinalidae Ridgway, 1901} \\
\hline Granatellus pelzelni Sclater, 1865 & polícia-do-mato & $g(2)$ & $\mathrm{mtf}$ & 25 & sr \\
\hline Caryothraustes canadensis (Linnaeus, 1766) & furriel & $g(2)$ & $\mathrm{mtf}, \mathrm{ca}$ & $17,18,24$ & s2, sr \\
\hline Cyanoloxia rothschildii (Bartlett, 1890) & azulão-da-amazônia & $g(1,2)$ & $\mathrm{mtf}$ & $4,15,17,18,21,22,24$ & $\mathrm{aw}, \mathrm{sr}$ \\
\hline \multicolumn{6}{|l|}{ Fringillidae Leach, 1820} \\
\hline Euphonia plumbea Du Bus, 1855 & gaturamo-anão & $\vee(1,2)$ & $\mathrm{ca}$ & 4 & aw, s2 \\
\hline Euphonia chlorotica (Linnaeus, 1766) & fim-fim & $\circ(2), v(2)$ & ig, ca & 9 & s2 \\
\hline Euphonia chrysopasta Sclater \& Salvin, 1869 & gaturamo-verde & $g(2)$ & $\mathrm{mtf}$ & 21 & $\mathrm{sr}$ \\
\hline Euphonia rufiventris (Vieillot, 1819) & gaturamo-do-norte & $g(2), v(1), o(2)$ & $\mathrm{mtf}$, ig & $4,15,17-20,22$ & aw, sr \\
\hline
\end{tabular}

'Evidences: c (voucher specimens with identification numbers in the bird collection of the Instituto Nacional de Pesquisa da Amazônia - INPA under care of Dr. Mario Conh-Haft), f (photographs), g (tape recordings), o (observations without documentation), $v$ (species identified by vocalizations without documentation). Numbers following the evidence referred to following authors: (1) Andrew Whitaker, (2) Sérgio Borges, (3) Ricardo Almeida, (4) other collaborators: a) Carlos Durigan, b) Zig Kock, c) Marcelo Moreira, d) Cintia Cornelius.

${ }^{2}$ Habitats: ca (white sand campinas and campinaranas), cal (altitudinal fields), $\mathrm{cp}$ (secondary forests and other human-disturbed habitats), ig (igapó flooded forests), $\mathrm{mtf}$ (terra firme forests), $\mathrm{mm}$ (montane forests), $\mathrm{r}$ (rivers, lakes or streams).

${ }^{3}$ Sites: Numbers referred the sites show in the figure 1 . Number 1 identify random observations for which was not possible to assign a geographic coordinate and includes records made during dislocation between mainly studies sites. Following geographic coordinates of each site indicated in figure 1: 2 (-63 26 46/0 55 03), 3 (-63 25 10/0 28 05), 4 (-62 58 53/0 19 24), 5 (-63 15 31/0 27 19), 6 (-63 24 24/0 24 19), 7 (-63 14 35/0 3407$), 8$ (-63 25 43/0 28 30), 9 (-63 28 16/0 28 40), 10 (-63 15 32/0 21 28), 11 (-63 15 47/0 19 26), $12(-632653 / 05256), 13$ (-63 27 11/0 52 06), 14 (-63 $2735 / 05216), 15$ (-62 44 09/-0 18 50), 16 (-62 48 13/0 14 08), 17 (-63 27 13/0 51 59), 18 (-63 28 13/0 52 40), 19 (-63 11 01/0 0832$), 20$ (-62 54 08/0 24 46), 21 (-62 44 39/0 16 35), 22 (-62 47 53/0 20 13), 23 (-63 23 02/0 25 08), 24 (-63 28 08/0 51 56), 25 (-62 44 36/0 16 10), 26 (-62 47 47/0 19 07).

${ }^{4}$ Sources: aw) Andrew Whittaker, December 2004, s1) Sérgio Borges, July 2006, s2) Sérgio Borges July/August 2007, sr) Sérgio Borges and Ricardo Almeida, August 2008, sc) Sérgio Borges, Cíntia Cornelius and Claudeir Vargas July 2010, ter) Carlos Durigan, Zig Kock, Marcelo Moreira. 
Appendix S1. Pictures of noteworthy bird species recorded in the Aracá region, Northwestern Brazilian Amazon.

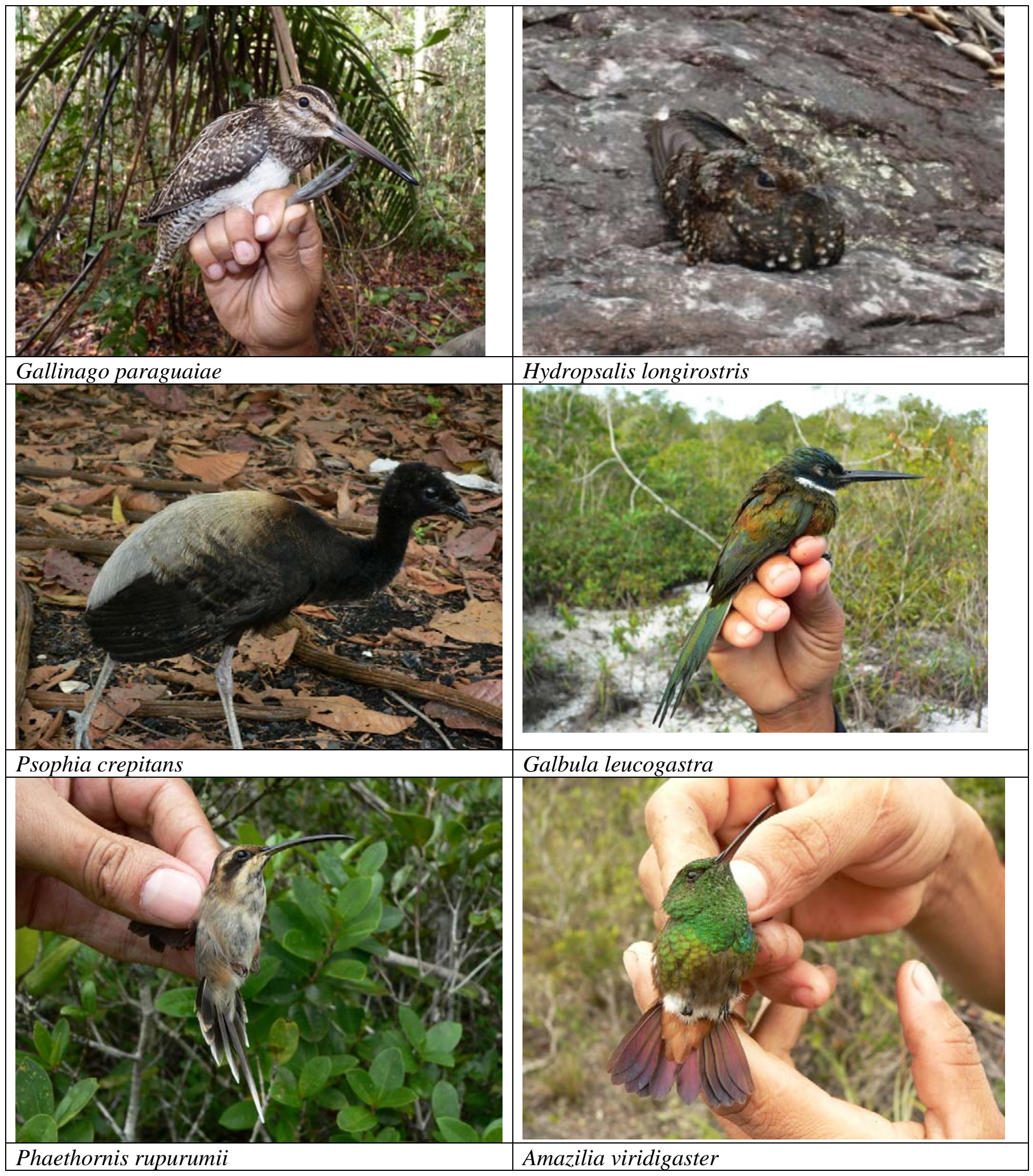




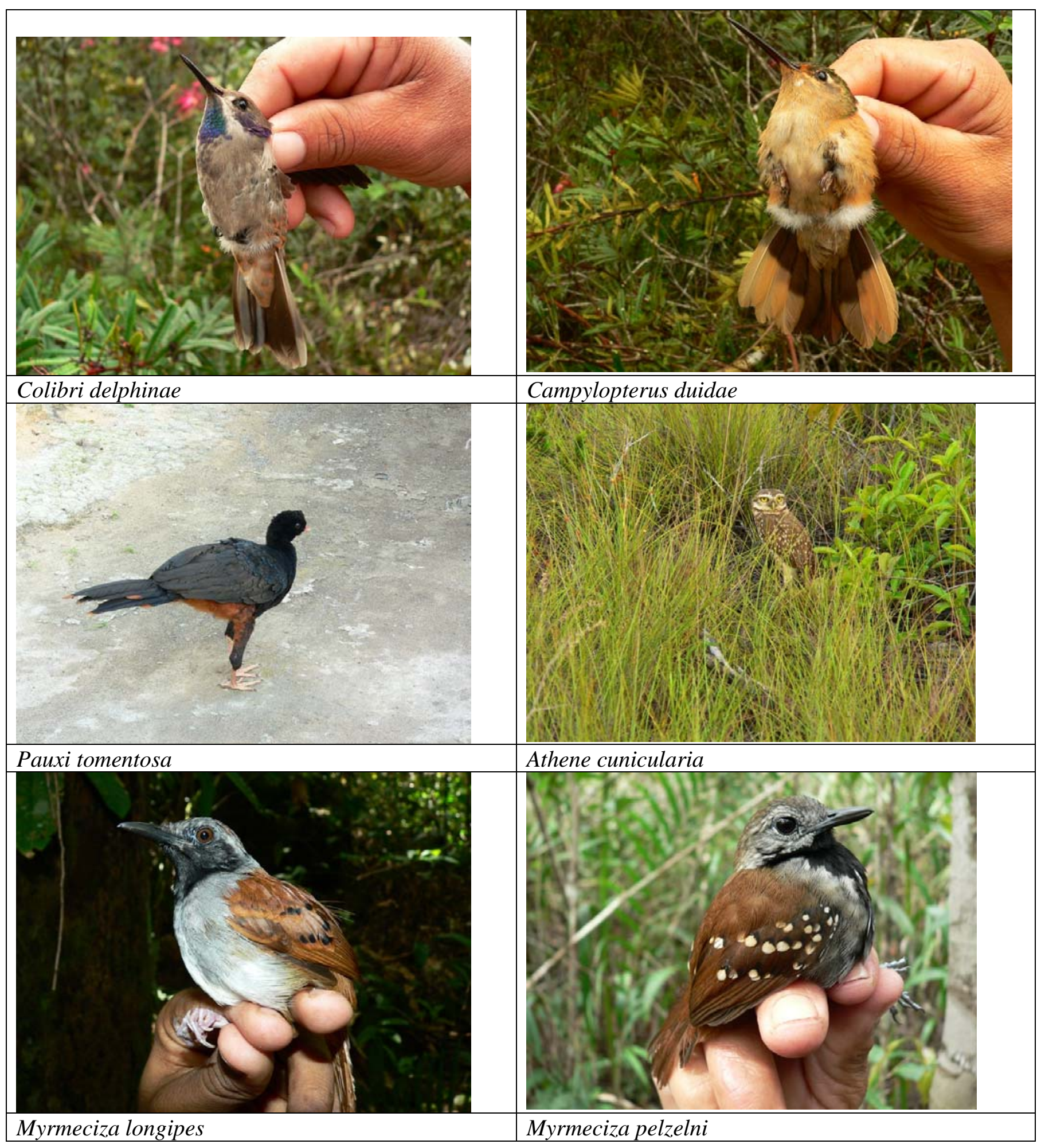




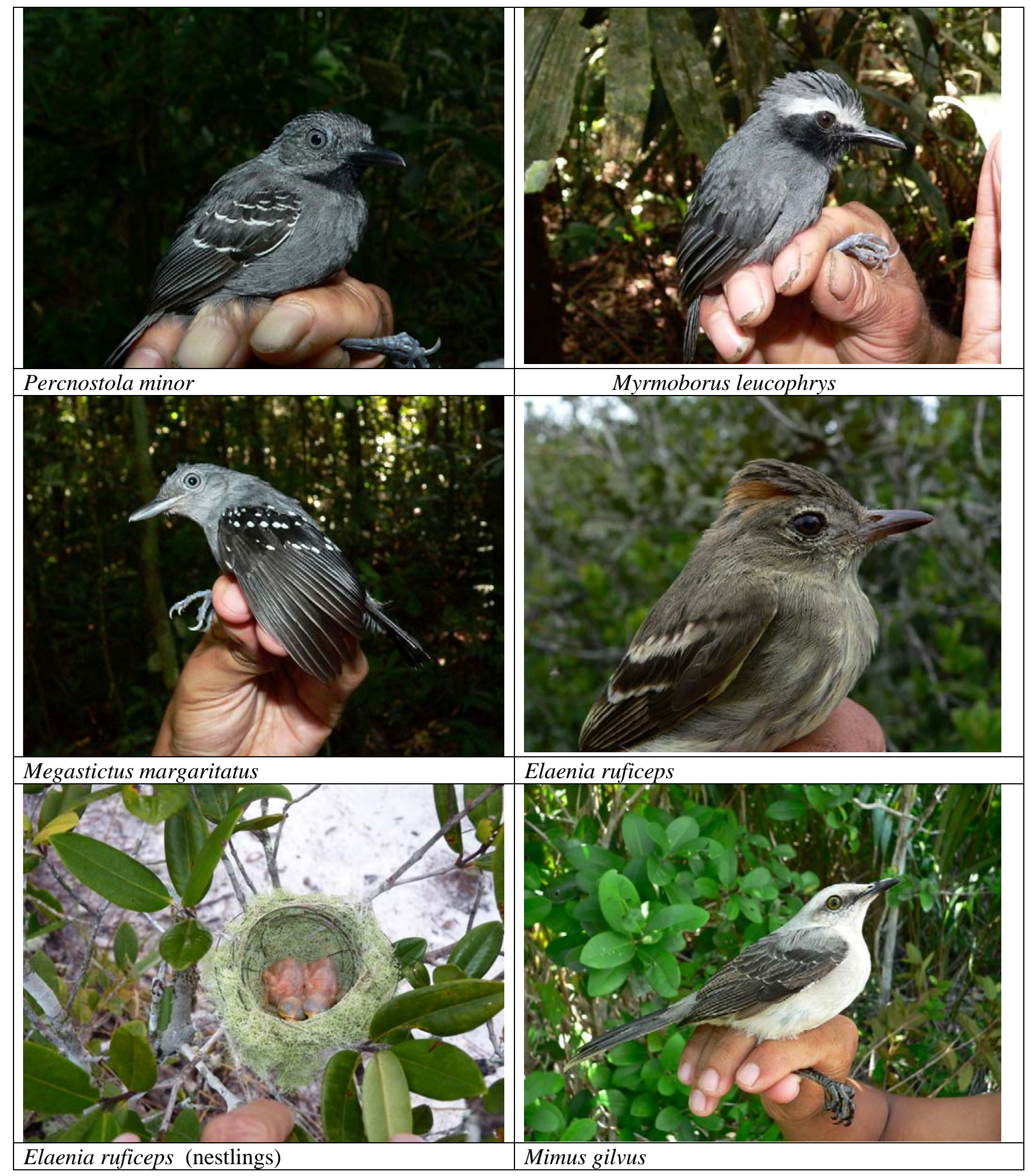




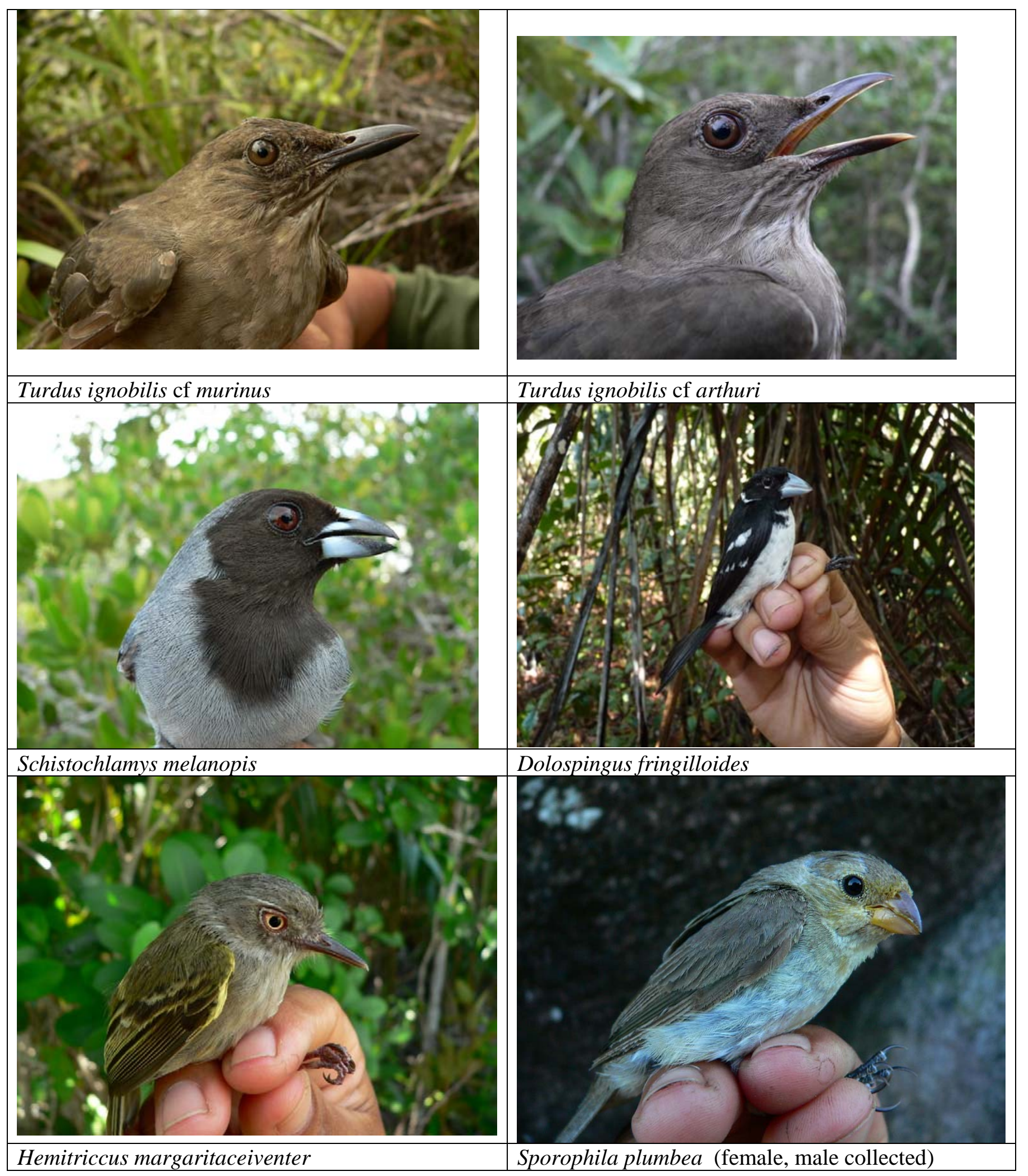




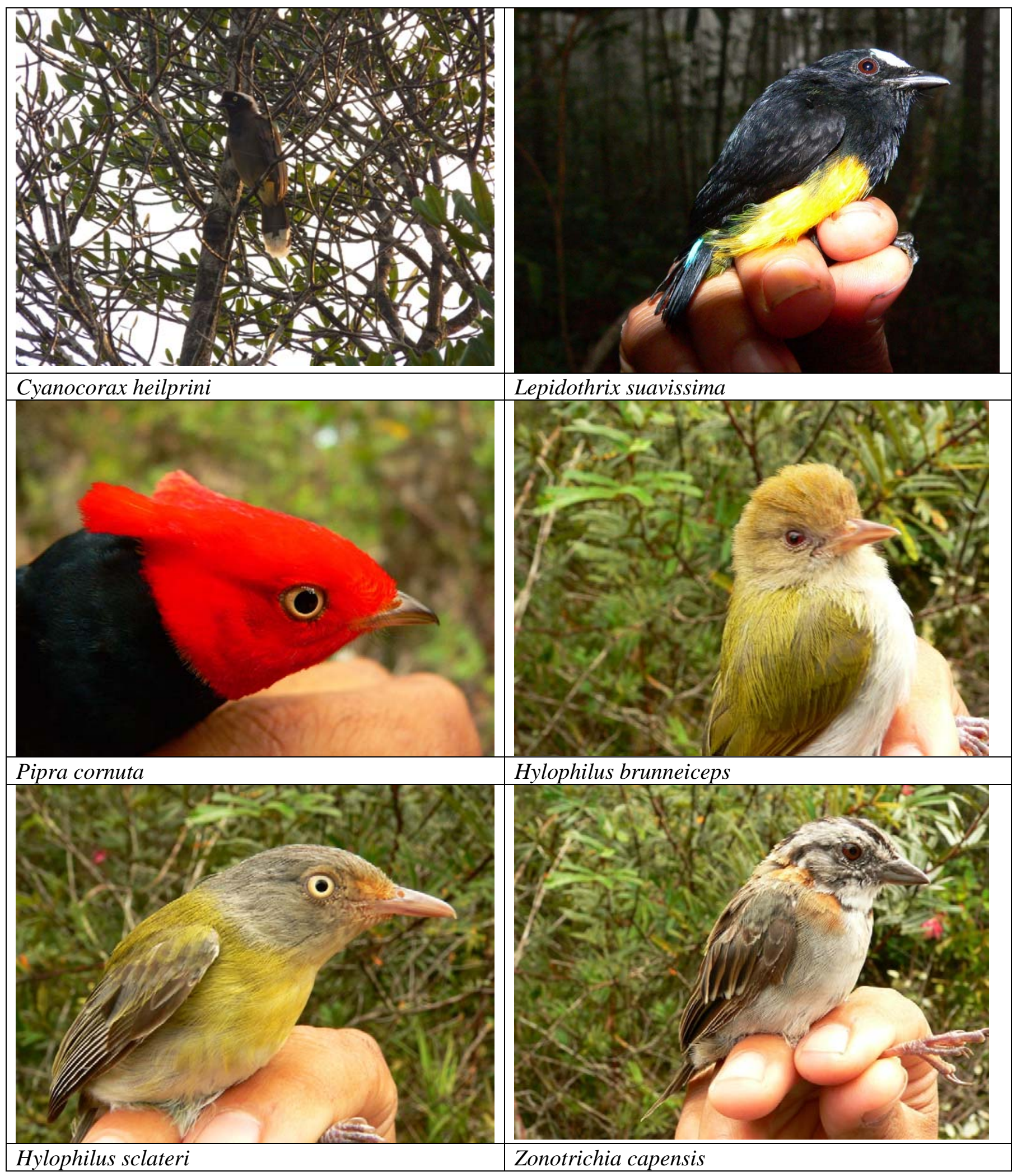

